\title{
XXII. Hans Dieter Beck in der Berliner Republik 1990-2013 \\ Die Expansion wird noch größer
}

\section{Das Ende der DDR und die Folgen für den Verlag}

D er Fall der Berliner Mauer war gleichzeitig das Signal für die westdeutsche Wirtschaft, in Ostdeutschland nach Betrieben zu suchen, an denen man sich beteiligen oder sie möglichst übernehmen könne. Denn es war klar, dieser Fall war auch ein Zeichen des Zusammenbruchs der sozialistischen Ökonomie, die dringend Kapital brauchte, und das hieß: westliches Kapital. So begab sich C.H.Beck auf den Weg nach Ostberlin zum Staatsverlag der DDR. In ihm erschien praktisch die gesamte juristische Literatur des Landes, Textausgaben, Kommentare, Lehrbücher. Aber die Münchner kamen zu spät. Der Freiburger Verlag Rudolf Haufe war schon vor der Wende da und hatte danach seine Zusammenarbeit mit den Ostberlinern vertieft. Deshalb beschloss der Beck-Verlag, von München aus ein eigenes juristisches Programm für Ostdeutschland aufzubauen, zunächst aus den Quellen der DDR, was auch deshalb notwendig wurde, weil nach den Wahlen zur Volkskammer im März 1990 dieses Parlament begann, das dortige Rechtssystem in freiheitlicher Art umzugestalten und an das Recht der Bundesrepublik anzupassen. Wichtige Grundlage dafür war die Vereinbarung einer Währungs-, Wirtschafts- und Sozialunion der beiden Länder, wobei man noch damals überwiegend, davon ausging, dass die DDR zunächst weiter ein eigenständiger Staat bleiben werde. Dies war auch die Einschätzung im Verlag.

Aufgrund dieser Zusammenhänge wurde Anfang 1990 in zwei Richtungen nachgedacht, nämlich ein eigenes Programm zum Recht der DDR zu entwickeln und Bücher zum bundesdeutschen Recht heraus zu bringen, die es Juristen in der DDR ermöglichen sollten, sich schnell in die Grundlagen der westdeutschen Rechtsordnung einzuarbeiten.

So ging es zunächst darum, zuverlässige Textausgaben zum Recht der DDR herauszubringen. Das war manchmal gar nicht so einfach. Für die volkseigene Wirtschaft hatte ein Vertragsgesetz gegolten, das der Umsetzung von Planauflagen in den Betrieben und im Rechtsverkehr unter ihnen gedient hatte. Mit der Umwandlung in privatrechtliche Unternehmen wa- 
ren die planwirtschaftlichen Rechtsgrundlagen hinfällig geworden. Stattdessen galten nun das HGB, das GmbH-Gesetz und das Aktiengesetz, die in der DDR nie aufgehoben waren. Aber sie hatten dort noch die Fassung des Jahres 1945, weil sie die DDR nicht geändert hatte. Nur wo holt man gerade auf die Schnelle diese Gesetzestexte her? Aber angesichts der Verhältnisse, die in den ersten Monaten des Jahres 1990 in der DDR herrschten, war es schon ein Problem, die zahlreichen Gesetze, die die Volkskammer nun zum Umbau des Staatswesens produzierte, schnell zu erhalten und dazu gar in München. Noch funktionierte die Post nur mit großen Verzögerungen und ob die Staatsdruckerei der DDR zuverlässig einen Versand der Gesetzblätter organisiert hätte, war mehr als fraglich. Aber man fand heraus, dass die Staatsdruckerei einen Laden für DDR-Gesetzblätter in Berlin Mitte eröffnet hatte. So stellten sich dann auch Beck'sche Lektoren in die Schlange und erstanden jeweils die neuesten Gesetze der DDR-Volkskammer.

So entstanden nicht nur kleine rote Beck'sche Textausgaben etwa zum Handelsrecht, Gesellschaftsrecht und Arbeitsrecht. Vielmehr gab es bereits im Sommer 1990 nach westdeutschem Vorbild auch einen «DDRSchönfelder» und einen «DDR-Sartorius», in denen die maßgeblichen Rechtsquellen zusammengefasst waren, die nach westdeutschem Verständnis dem Zivil- und Strafrecht bzw. dem öffentlichen Recht zuzuordnen sind.

Kurze Darstellungen zum Recht der DDR konnten nur noch in begrenztem Umfang erscheinen. Gleiches gilt für Einführungen in das bundesdeutsche Recht, die speziell für DDR-Juristen konzipiert wurden. Immerhin kam aber das von Rechtsanwalt Burkhard Messerschmidt herausgegebene Hand- und Schulungsbuch »Deutsche Rechtspraxis» auf den Markt und der von Othmar Jauernig herausgegebene BGB-Kommentar erschien in broschierter Form besonders preisgünstig.

Die deutsche Wiedervereinigung kam dann doch viel schneller als gedacht. Im August 1990 hatte DDR-Ministerpräsident de Maizière Bundeskanzler Kohl an dessen Urlaubsort am Wolfgangsee dargelegt, die Volkskammer werde den Beitritt der DDR zum Bundesgebiet erklären, wenn es bis zum Oktober 1990 keine gesamtdeutschen Wahlen gebe. Die wirtschaftliche Lage in der DDR habe sich derart dramatisch zugespitzt, dass er keinen anderen Ausweg sehe. Am 31. August 1990 wurde dann bereits der Einigungsvertrag zwischen beiden deutschen Staaten geschlossen, der die staatliche Einheit zum 3. Oktober 1990 festsetzte. Damit trat von einem Tag auf den anderen praktisch das gesamte Recht der DDR außer Kraft. Nur wenige Randbereiche galten - zumeist nur übergangsweise - 


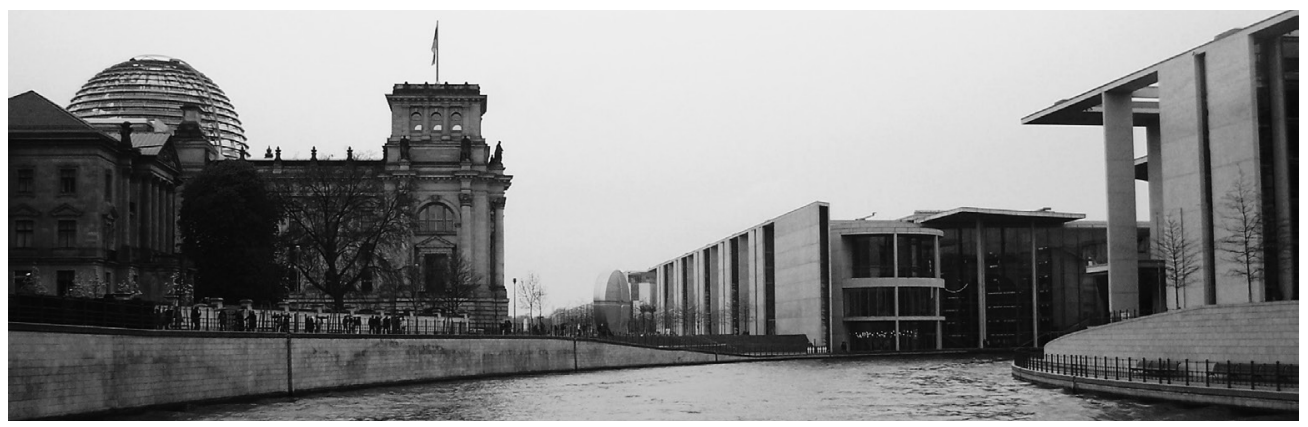

Berlin wird Hauptstadt. 1999 nehmen Regierung und Parlament dort ihre Arbeit auf. Links das Reichstagsgebäude mit der Kuppel von Norman Foster, rechts das Marie-Elisabeth-LüdersHaus von Stephan Braunfels, in der Mitte der Spreebogen.

als Bundes- oder Landesrecht weiter. Im Übrigen aber trat bundesdeutsches Recht in Kraft.

Nun mussten Rechtsanwälte und Notare, Gerichte, Behörden, Universitäten und Unternehmen mit Literatur zum bundesdeutschen Recht versorgt werden. So hatte sich der Verlag darauf zu konzentrieren, im Gebiet der neuen Bundesländer einen schlagkräftigen Vertrieb aufzubauen und seine Werke nachzudrucken oder höhere Auflagen einzuplanen. Literatur zum DDR-Recht gab es dagegen praktisch nicht mehr. Eine gewisse Bedeutung hat lediglich die Erläuterung der zunächst noch fortgeltenden Gesamtvollstreckung gespielt, die etwa im von Karsten Schmidt fortgeführten Kurz-Kommentar «Insolvenzgesetze», oder im von Peter Gottwald herausgegebenen Insolvenzrechts-Handbuch erfolgte.

Andere Bereiche des DDR-Rechts hat nach 1990 erst der Bundesgesetzgeber geregelt. So konnte in der DDR über volkseigenen Grund und Boden nicht verfügt werden. Um Bürgern der DDR dennoch die Möglichkeit zu eröffnen, Eigenheime zu errichten, erhielten sie am Grundstück ein Nutzungsrecht. Losgelöst vom Eigentum am Grundstück konnte der «Häuslebauer» Gebäudeeigentum an seinem Eigenheim erwerben, das seinerseits verkehrsfähig war. Mit der deutschen Einheit sollte nun aber wieder die Einheit von Grundstück und Gebäude als dessen wesentlicher Bestandteil festgelegt werden. Die damit zusammenhängenden Probleme hat der Gesetzgeber 1992 im Sachenrechtsbereinigungsgesetz geregelt und dazu erschienen nunmehr maßgebliche Kommentare im Verlag C.H.Beck: Vossius, Sachenrechtsbereinigungsgesetz, 2. Auflage 2oo6; Prütting/Zimmermann/ Heller, Grundstücksrecht Ost, 2003 (Loseblatt) und Busche u. a., Neues Schuld- und Sachenrecht im Beitrittsgebiet, ein 1997 als seperate Kom- 
mentierung aus dem Münchener Kommentar zum BGB entnommener Sonderband, diesmal freilich in "grauer» Austattung.

Eine weitere Materie hat bis heute Bedeutung: Die Wiedergutmachung für das Unrecht, das unter SED-Herrschaft verübt worden ist. Sie besteht aus zwei unterschiedlichen Rechtszweigen. Der erste ist entstanden aus der Forderung der Bundesrepublik Deutschland gegenüber der DDR, die offenen Vermögensfragen zu regeln, die wegen Enteignungen von Bundesbürgern und in das Bundesgebiet geflohenen DDR-Bürgern entstanden sind und die im Grundlagenvertrag von 1972 ausdrücklich offen gelassen worden sind. Deshalb heißt dieser Rechtszweig «Recht der offenen Vermögensfragen.» Der andere Rechtszweig geht auf eine Initiative des «Runden Tischs» in der DDR zurück, dem es nach dem Vorbild erster Regelungen in der UdSSR unter Staatspräsident Michail Gorbatschow insbesondere darum ging, DDR-Bürger, die verfolgt worden waren, weil sie von ihren verfassungsrechtlichen Rechten Gebrauch gemacht hatten, zu rehabilitieren. Daraus hat sich das Rehabilitierungsrecht entwickelt.

Schon weil das Recht der offenen Vermögensfragen erst mit dem Einigungsvertrag verabschiedet werden konnte, hat es seine ursprüngliche Funktion, vermögensrechtliche Ansprüche von Bundesbürgern gegenüber der DDR durchzusetzen, geändert. Es gilt vielmehr für alle entschädigungslosen, diskriminierenden Vermögensschädigungen, ohne dass sie mit weitergehendem Unrecht in Verbindung stehen.

Die erst 1992 und 1994, erlassenen Rehabilitierungsgesetze gelten dagegen für die Fälle der straf- oder verwaltungsrechtlichen politischen Verfolgung. Solche Maßnahmen haben etwa bestanden aus perfiden Drangsalierungen durch die Stasi, politischen Schauprozessen mit drakonischen Strafen oder systematischer Ausschaltung von Unternehmern und Großgrundbesitzern als Klassenfeinde aus der Gesellschaft durch repressive Methoden unter schwerster Missachtung elementarer strafprozessualer Garantien nach dem Vorbild der in der UdSSR unter Stalin praktizierten Vertreibung der Kulaken und der stalinistischen Säuberungen. Gegenüber den bloßen Enteignungen, die das Recht der offenen Vermögensfragen erfasst, war die politische Verfolgung grundsätzlich das schlimmere Unrecht. Dennoch hat das Recht der offenen Vermögensfragen bei der Aufarbeitung des SED-Unrechts die wesentlich größere Rolle gespielt. Das bedeutete auch, dass der Verfolgungscharakter von Repressionsmaßnahmen in Tausenden Fällen bis heute nicht erkannt und rehabilitiert ist. Deshalb hat insbesondere eine Aufarbeitung der Massenrepressionen im Rahmen der 


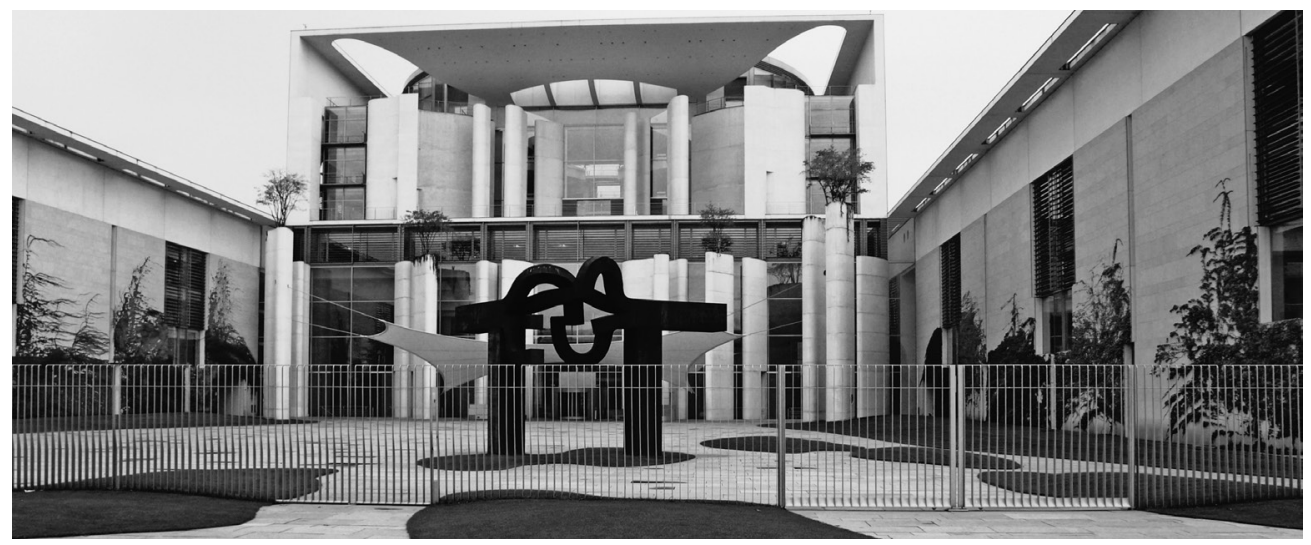

1999 nahm die Bundesregierung ihre Arbeit provisorisch im ehemaligen Staatsratsgebäude der DDR auf. 2001 wurde das neue Kanzleramt bezogen (Architekt Axel Schultes).

missverständlich als «Boden- und Wirtschaftsreform» bezeichneten Verfolgungsaktionen bislang nicht stattgefunden.

Das wesentlich größere Interesse am Recht des offenen Vermögens hat sich auch in der Literatur des Verlags niedergeschlagen. Das Vermögensgesetz ist gleich in drei Kommentaren erläutert. Schwerpunktmäßig die Sicht des Bundesministeriums der Justiz vermittelt der von Gerhard Fieberg und Harald Reichenbach, den beiden Referenten des Gesetzes, herausgegebene Loseblatt-Kommentar. 1995 hat Franz Jürgen Säcker einen gebundenen «grauen» Kommentar «Vermögensrecht» herausgegeben, der die materiellrechtlichen Vorschriften des Vermögensgesetzes, bemüht um wissenschaftliche Ausgewogenheit, erläutert. Da das Werk aber keine Neuauflage erlebt hat, informiert es nur über die Frühzeit des Rechts der offenen Vermögensfragen. Mit rund 2500 Seiten ist der Kommentar des Vermögensgesetzes, verfasst von Johannes Wasmuth, Werner Wellhöfer, Kurt Kiethe, Kay Windthorst und Hannes Flotho die umfangreichste Erläuterung des Gesetzes. Sie ist Teil des auf vier Bände ausgelegten «Rechtshandbuchs Vermögen und Investitionen in der ehemaligen DDR» und legt das Gesetz in der Tendenz in einer für die Geschädigten eher günstigen Weise aus. Dazu setzt sie sich immer wieder kritisch mit der Rechtsprechung auseinander, wenn diese eine zum Nachteil der Betroffenen betont restriktive Linie verfolgt.

Und das Rehabilitierungsrecht? Hier ist im Verlag nur ein Torso erschienen, das Grundwerk «Rehabilitierungsrecht» von Wolfgang Pfister und Wolfgang Mütze. Selbst die zentralen Vorschriften zum Anwendungsbereich der Rehabilitierungsgesetze sind dort nicht erläutert. Und mehr noch: Nicht 
einmal eine Ergänzungslieferung hat dieses Werk bislang erlebt. Auch das mag ein Grund dafür sein, dass die Aufarbeitung des SED-Unrechts in durchaus beträchtlichem Umfang mißlungen ist. Immerhin standen den Gerichten so nur kleinere Kommentare, einer zum Strafrechtlichen, ein anderer zum Verwaltungsrechtlichen Rehabilitierungsgesetz aus anderen Verlagen zur Verfügung, die bereits seit langem veraltet sind.

\section{Aufbruch ins östliche Mitteleuropa}

\section{a) Verlagsgründung in Warschau}

In Polen gab es ab dem Jahresbeginn 1992 zunächst eine recht gründliche Sondierungsphase mit dem Ziel auszuloten, wie die Marktlage und die Autorenszene beschaffen waren, ob ein deutsches Verlagshaus mit geschichtlich begründeten Ressentiments zu rechnen hätte und ob eine Übernahme bzw. eine Kooperation mit einem etablierten polnischen Verlag in Betracht kamen. So führte man unter anderem Gespräche mit einem wenig für Neuerungen aufgeschlossenen Leiter des «Juristischen Staatsverlags», einem monopolartig agierenden Relikt aus der kommunistischen Ära. Solcherart Verhandlungen führten schließlich zu der Erkenntnis, dass eine Eigengründung vermutlich der bessere Weg sein würde.

In der Folgezeit schickte Hans Dieter Beck seinen Assistenten, der sich mittels polnischer Familienbeziehungen und einiger Sprachkenntnisse rasch Kontakte erschließen konnte, vielfach auf Sondierungsreisen nach Polen. Wertvolle Unterstützung leistete dabei Professor Władysław Rozwadowski, der so manche namhafte Autorenpersönlichkeit zu einem ersten Orientierungsgespräch an den Tisch zu holen vermochte. In diesen Treffen an den rechtswissenschaftlichen Fakultäten in Warschau, Posen, Krakau und Danzig wurde nicht nur offenbar, dass die befürchtete Reserviertheit gegenüber einem deutschen Verlagshaus dort gar nicht existierte, sondern es wurde - ganz im Gegenteil - zumeist reges Interesse an einer Zusammenarbeit mit dem in Polen durchaus bekannten und geschätzten Beck Verlag bekundet. So entstanden bereits im Sommer 1992 erste konkrete Projekte und sogar Verlagsverträge für den seinerzeit noch gar nicht existenten Verlag «Beck Polska».

Unbedingt erwähnenswert ist aus dieser Phase das Projekt des «polnischen Schönfelders», für das mit Professor Zbigniew Radwański eine in jeder Hinsicht herausragende Autorität des polnischen Zivilrechts als Herausgeber gewonnen werden konnte. Radwański, der neben seiner Pro- 
fessur in Posen auch den Vorsitz im «Obersten Gesetzgebungsrat» innehatte und zweifellos die Bedeutung dieses Projekts erkannte, war davon überzeugt, dass die erstmalige Konsolidierung der 120 wichtigsten Gesetze des Landes nur mit Hilfe einer ganzen Mannschaft namhafter Experten gelingen konnte. So versammelte er kurzerhand noch sechs weitere Professoren um sich, jeder von ihnen ein Spezialist in einer derjenigen Einzeldisziplinen, die in ihrer Summe eben den «Schönfelder» ausmachen.

Für die konkrete Umsetzung war nun zusätzliche Unterstützung vonnöten, und so sandte Hans Dieter Beck auch zwei seiner Münchener Lektoratsleiter, um das hochkarätig besetzte Redaktionskomitee im Umgang mit den ungewohnten Beck'schen Redaktionsrichtlinien anzuleiten. Tatsächlich war die allgemeine Versorgungslage im Lande damals noch so schlecht, dass die Münchener nicht nur Berge von Fotokopien aus dem polnischen Gesetzblatt, sondern auch Scheren und Klebstifte für das Erstellen konsolidierter Texte und sogar Buntstifte zum redaktionellen Auszeichnen mit nach Polen brachten. So ausgestattet konnte dann die Manuskriptarbeit für den 24,00 Seiten starken Loseblattband «Polskie Ustawy» (Polnische Gesetze) wirklich beginnen.

Unter den geschilderten Umständen scheint es doch bemerkenswert, dass das Werk nach nur neun Monaten intensiver Arbeit im Mai 1993 pünktlich zur Warschauer Buchmesse erschienen ist. Dabei war der Druck seinerzeit noch in der Nördlinger Druckerei zu erledigen, denn an eine Verarbeitung von Dünndruckpapier und an eine präzise Lochung des Buchblocks war damals in Polen noch nicht zu denken. Dank gezielter Streuung eines Prospekts als Beilage zum amtlichen Gesetzblatt konnte Beck beinahe im Handumdrehen 5000 zahlende Abonnenten gewinnen und mit diesen ersten Einnahmen den weiteren Aufbau eines polnischen Verlagsprogramms zu einem guten Teil finanzieren. Dieser Bilderbuchstart hat sich für «Polskie Ustawy» und die wenig später gegründeten parallelen Loseblattwerke leider nicht sehr lange fortgesetzt, denn ab den späten neunziger Jahren war die Flut der Reformgesetzgebung derart ausgeufert, (es gab Jahre mit 27 umfangreichen Ergänzungslieferungen), dass drastische Einbrüche bei den Bezieherzahlen nicht ausbleiben konnten.

Um aber in der Chronologie zu bleiben, sind noch ein paar Ereignisse rund um die formelle Verlagsgründung nachzutragen. Bis in den Herbst des Jahres $199^{2}$ war Hans Dieter Beck zu der Einschätzung gelangt, dass die Idee eines Engagements in Polen genügend vielversprechend ist, und so wurde alsbald ein erster polnischer Verlagsleiter eingestellt. Im März 1993 wurde unter der Firma «Wydawnictwo C. H. Beck sp. z o. o.» eine 
GmbH polnischen Rechts in das Warschauer Handelsregister eingetragen und etwa zur selben Zeit bezog eine kleine Verlagsmannschaft ihr erstes Büro am Rande der Warschauer Innenstadt.

Auf diese Weise konnte zwar die Programmarbeit weiter vorangetrieben, aber keineswegs ein effektiver Vertrieb geleistet werden. Um die Lösung dieses Problems kümmerte sich der Vertriebsleiter des Münchener Mutterhauses und fand alsbald eine kluge Lösung. Der damals noch staatliche Wissenschaftsverlag «PWN» war bereit, zu kooperieren und gegen eine umsatzabhängige Provision den Verkauf aller Beck'schen Publikationen über sein breites Verkaufsnetz zu besorgen. Als dann zeitgleich mit dem «polnischen Schönfelder» auch die ersten Bücher (zwei Lehrbücher und vier broschierte Textausgaben) sowie bald darauf die erste Monatszeitschrift «Monitor Prawniczy» erschienen, hatte der kleine Beck Verlag schon vom Start weg eine flächendeckende Buchhandelspräsenz.

In dem anfangs sehr instabilen polnischen Markt waren rasche Veränderungen beinahe an der Tagesordnung. So musste man sich von dem zunächst gefundenen Verlagsleiter recht bald wieder trennen, fand dann aber bald stabilen Ersatz für diesen wichtigen Posten. Ungeachtet solcher Erschütterungen ging der Programmaufbau zügig voran. Vor allem die Kommentarliteratur, die schon in der polnischen Nachkriegszeit eine gewisse Tradition entwickelt hatte, spielte eine erhebliche Rolle für das steile Umsatzwachstum der ersten Jahre. Gleich zum Einstieg gelang hier ein echter Bestseller, indem mit dem zweibändigen HGB-Kommentar von Sołtysiński, Szajkowski und Szwaja die erste Neukommentierung des polnischen Gesellschaftsrechts seit den 1930er Jahren publiziert werden konnte. Um dieses bis heute führende Werk herum entstand ein recht breit gefächertes Programm von Kommentaren unterschiedlicher Umfänge und Preislagen, allen voran die jeweils mehrbändigen Werke zum Zivilgesetzbuch (herausgegeben von Pietrzykowski) und zur Zivilprozessordnung (Piasecki). Im Jahre 2006 konnte der Verlag mit dem ersten einbändigen Kommentar zum Zivilgesetzbuch (Gniewek) bereits die vierte parallele Kommentar-Reihe eröffnen. Wenn mit diesem Werk auch vielleicht die Hoffnung auf einen künftigen «Palandt» verbunden war, so durfte man dies doch nicht offen so aussprechen, denn das polnische Wort palant bedeutet «Dummkopf».

Dank einer bemerkenswerten Experimentierfreude konnte der polnische Verlag inzwischen eine wohl landesweit führende Titelvielfalt aufbauen, die von einer ausgesprochen breiten Basis namhafter Autoren getragen wird. Neben den Kommentaren prägen heute vor allem Textausgaben, 
Lehrbücher, Handbücher und Formularwerke, Fachlexika und nicht zuletzt Zeitschriften das Beck'sche Erscheinungsbild in Polen. Neben diesem «klassischen» Auftritt eines juristischen Fachverlages wurde ab dem Ende der 1990er Jahre dann auch noch in anderer Hinsicht Neuland beschritten: Unter dem Logo «Beck Info Biznes» veröffentlicht Beck in Polen seitdem auch ratgeberartige Werke für Nicht-Juristen in den Themenbereichen Personalwesen und Finanzen.

Wenn Beck einen Faktor in der Entwicklung der mittel- und osteuropäischen Märkte unterschätzt hatte, so war es der wahrhaft kometenhafte Aufstieg der elektronischen Medien und ihrer sofortigen Akzeptanz unter den Juristen dieser Länder. Im Nachhinein betrachtet liegt es vielleicht nahe, dass ab der postkommunistischen Zeitenwende zunächst einmal junge und jüngste Juristen auf der beruflichen Erfolgswelle schwimmen, dass also Menschen mit hoher Affinität zum Computer das Käuferverhalten viel stärker bestimmen als hierzulande. Mehr noch ist es aber der unablässig galoppierenden und reichlich unübersichtlichen Gesetzgebung in den Reformstaaten zuzuschreiben, dass die meisten Juristen arg verunsichert nach Orientierung und Sicherheit suchen und ihr Heil in tagesaktuellen elektronischen Gesetzgebungsdatenbanken finden. Die Bedeutung derartiger Datenbanken wurde in Polen mit Produkten namens «Lex» und «Lex Polonica» gegen Ende der Neunzigerjahre deutlich spürbar und verschaffte den multinationalen Verlagsgruppen Wolters Kluwer und LexisNexis (damals: Reed Elsevier) einen späten, aber wirksamen Einstieg in den polnischen Markt. Beide Konzerne konnten mehrere lokale Firmen und Produkte übernehmen und diese mit vermutlich hohem finanziellem Aufwand zu hohen Umsatzzahlen entwickeln.

Gegen diese neuen Wettbewerber musste auch Beck sich plötzlich sehr viel konsequenter auf elektronische Publikationsformen konzentrieren und mit einigen Investitionen ein ebenbürtiges Konkurrenzprodukt schaffen. Während einer zweijährigen Vorlaufphase wurden also Dutzende von Jahrgängen des Gesetzblattes digitalisiert und konsolidiert, es wurde eine sowohl für CD-ROM-Produkte als auch für den Online-Betrieb geeignete Endanwender-Software entwickelt und es wurde nicht zuletzt die Arbeitsweise des Lektorats auf die zeit- und kostensparende medienneutrale Datenhaltung umgestellt. Das Ergebnis dieser Kraftanstrengungen wurde dann schließlich im Jahre 2002 unter dem Produktnamen «Legalis» gestartet.

Insoweit ähnlich dem deutschen Vorbild «beck-online» ist auch Legalis eine modular aufgebaute Rechtsdatenbank mit getrennt beziehbaren Abon- 
nements zu den verschiedenen Arbeitsgebieten der juristischen Praxis. Besonderheiten des polnischen Marktes bedingen jedoch einige Unterschiede zu «beck-online». So sind in der polnischen Datenbank Gesetzgebung und Rechtsprechung zu sämtlichen Rechtsgebieten in einem einheitlichen «Basismodul» vereinigt. Zudem bevorzugen bis heute noch viele der Datenbanknutzer in Polen eine Offline-Version auf DVD, die deshalb alternativ zur Online-Lösung angeboten wird. Der späte Markteinstieg im elektronischen Publizieren war für Beck Warschau eine mühsame Angelegenheit und ist es im Grunde bis heute, denn gegen die jahrelange Gewöhnung der Kunden an das zuerst etablierte Produkt der Konkurrenz lässt sich nur ganz allmählich Boden gutmachen. Dass dies offenbar dennoch gelingt, dürfte in hohem Maße dem Ansehen der Beck'schen Kommentarwerke zu verdanken sein. Diese sind sehr konsequent in «Legalis» einbezogen und schaffen dort einen erheblichen Kaufanreiz. Damit zählt inzwischen auch Beck Warschau zu den Verlagen, die gerade auf elektronischem Gebiet rasches Wachstum verzeichnen können.

Die heutige Situation im polnischen Markt ist geprägt von einem angespannten Wettbewerbsverhältnis zwischen den drei Verlagen C.H.Beck, LexisNexis und Wolters Kluwer. Der Großteil des gesamten Marktvolumens im juristischen Publizieren entfällt in Polen schon auf elektronische Produkte. Dagegen tun sich Bücher schwerer als etwa in Deutschland nicht zuletzt auch wegen der Schnelllebigkeit der Gesetzgebung: diese lässt den Lebenszyklus einer jeden Neuauflage auf ein Minimum schrumpfen, was die Auflagenzahlen reduziert und neben den Herstellkosten auch den Aufwand für Vertrieb und Marketing in die Höhe treibt. Dieses Phänomen wird nicht zuletzt an dem eingangs erwähnten «polnischen Schönfelder» ersichtlich, der in den 20 Jahren seit der Verlagsgründung in Warschau sage-und-schreibe 180 Ergänzungslieferungen erlebt hat.

So sind derzeit rund 140 fest angestellte Mitarbeiter im Warschauer Beck Verlag darum bemüht, mit der Gesetzgebung Schritt zu halten, und produzieren jährlich etwa 350 Neuerscheinungen und Neuauflagen, rund 100 Ergänzungslieferungen und daneben diverse Zeitschriftenhefte, CD-ROMs, DVDs und Internetseiten. Mit dieser relativ breiten Marktpräsenz konnte Beck innerhalb eines überschaubaren Zeitraums eine anerkannte Marke schaffen. Dazu hat beigetragen, dass mitunter auf bewährte verlegerische Rezepte zurückgegriffen wurde, die auch in Polen guten Anklang fanden und nicht selten bald von anderen Verlagen übernommen wurden. Besonders freundliche Stimmen haben gar behauptet, der Einstieg von Beck in 
den polnischen Markt habe die gesamte juristische Szene des Landes spürbar bereichert. So betrachtet scheint die anfängliche Skepsis gegenüber dem Engagement in Polen heute eigentlich nur noch im Rahmen eines geschichtlichen Rückblicks erwähnenswert.

\section{b) Verlagsgründung in Prag}

Die Entwicklung in der Tschechischen Republik verlief in zeitlicher und weitgehend auch in sachlicher Hinsicht parallel zum Werdegang in Polen. Mit dem Unterschied, dass in Bezug auf die damals noch bestehende Tschechoslowakei eine Verlagsgründung eigentlich nicht in Frage kam. Da sich das juristische Verlagswesen auch heute noch fast ausschließlich in nationalen Märkten definiert, hängen die Erfolgsaussichten eines Markteintritts in erster Linie von der Bevölkerungszahl des jeweiligen Landes ab. In dieser Hinsicht war die Tschechoslowakei mit nur rund 15 Millionen Einwohnern weit weniger attraktiv als Polen mit seinen knapp 40 Millionen. Dabei war noch in keiner Weise absehbar, dass sich das Land wenige Jahre später auch noch teilen würde, so dass schließlich nur rund 10 Millionen Tschechen mit juristischer Literatur zu versorgen übrig blieben.

Aber auch hier kam wiederum dem «Schönfelder» eine wegweisende Bedeutung zu. Im März 1992 hatte die Lektoratskonferenz einen Publikationsvorschlag des Münchener Instituts für Ostrecht zu diskutieren, der auf die Schaffung einer Loseblattsammlung der wichtigsten tschechoslowakischen Gesetze abzielte. Kaum hatte man bei einer spontan anberaumten ersten Prag-Reise den dortigen Gesetzblattverlag «SEVT» als geeigneten Vertriebspartner ausfindig gemacht, machten sich zwei Münchener Lektoratsleiter an die Arbeit, die Gesetzessammlung «Československé Zákony» (später notgedrungen umbenannt in «České Zákony») aus den fotokopierten Archiven des Gesetzblatts Gestalt annehmen zu lassen. Ähnlich wie in Polen, so wurde auch hier wochenlang mit Schere, Kleber und Buntstiften gearbeitet, bis schließlich im Frühjahr 1993 ein leuchtend gelber Plastikordner unter dem schier unaussprechlichen Motto «Úplné znění» (bereinigte Fassungen) an rund 5000 zahlende Abonnenten innerhalb kürzester Zeit verkauft werden konnte.

Beflügelt von dem erfolgreichen Einstand wollte man gleich noch einmal nachsetzen und auch eine Zeitschrift nach dem Vorbild der NJW ins Leben rufen. Hierzu war es nun allerdings nötig, eine tschechoslowakische Gesellschaft zu gründen (so die Vorgabe des dortigen Presserechts) und vor allem auch eine Redaktion zu installieren. Auf dieser Basis ent- 
stand dann zeitgleich mit dem Start in Warschau auch in Prag bereits ein erstes kleines Verlagsprogramm und in dessen Mittelpunkt die so genannte «Právní Rozhledy» (Juristische Rundschau).

Sehr rasch wurde auch das Prager Verlagsprogramm um die klassischen Ingredienzien eines juristischen Verlagskatalogs ergänzt. Neben den ersten broschierten Textausgaben entstanden zunächst einige Lehrbücher, und der Verlag konnte dafür - ähnlich wie in Polen - recht rasch das Vertrauen der prominentesten Autoren gewinnen. Dabei war es immer auch entscheidend, so etwas wie einen Präzedenzfall zu schaffen: hatte sich erst einmal eine Persönlichkeit wie Professor Dušan Hendrych (u. a. mit seinem Lehrbuch zum Verwaltungsrecht) auf diesen neu gegründeten Verlag eingelassen, so war es anschließend entschieden leichter, kooperationswillige «Nachahmer» zu finden.

Ein weiterer Meilenstein für den Aufbau des Buchprogramms war sodann die Übernahme einer ganzen Reihe von Titeln vom Verlag «Frances». Diese konnte Beck für einen überschaubaren Betrag dort ablösen und bereits 1994, in aktualisierter Fassung und in neuer Aufmachung unter dem Beck'schen Greif auf den Markt bringen. Unter den so übernommenen Werken befanden sich vor allem etliche Kommentare, die zwar zu jener Zeit noch recht spärlichen Umfangs waren, aber im Laufe der Jahre und der späteren Neuauflagen zu stattlichen Büchern herangewachsen sind. So gründet sich das Ansehen des tschechischen Beck Verlages bis heute zu einem erheblichen Teil auf die in unverwechselbar graues Leinen gebundene «Edition kommentierter Gesetze» mit den Werken von Bureš/Drápal zur Zivilprozessordnung, von Švestka zum Bürgerlichen Gesetzbuch, von Štenglová zum Handelsgesetzbuch und einigen anderen mehr.

Der zunächst steile Aufschwung dieser Beck'schen Verlagsgründung wurde allerdings schon recht bald wieder flacher, denn ab Mitte der 199oer Jahre verloren die Loseblatt-Textausgaben, die zu Anfang den Programmaufbau weitgehend finanziert hatten, drastisch an Beziehern. Hinzu kam ab 1993 die Teilung des Staates in zwei fortan eigenständige Republiken, deren jeweilige Gesetzgebung sich immer weiter voneinander entfernte. Aus diesem Grund konnte das Prager Programm plötzlich nur noch zwei Drittel der ursprünglichen Bevölkerung erreichen, und ein ohnedies schon relativ kleiner Markt mit geringer Kaufkraft war über Nacht noch einmal zusätzlich geschrumpft.

In den nun folgenden Jahren wurde das juristische Programm Schritt für Schritt weiter komplettiert und es wurden angesichts des grundsätzlich schmalen Absatzmarktes mehrere Versuche unternommen, die Pro- 
gramm- und Umsatzbasis des Verlages zu erweitern. Unter diesen Initiativen, die leider nicht sämtlich erfolgreich verliefen, sei hier immerhin der Start eines wirtschaftswissenschaftlichen Buchprogramms erwähnt. Mit diesem hat sich C.H.Beck Prag ein zusätzliches (freilich viel kleineres) Fachsegment erschließen können, in dem mit Miloslav Synek, Robert Holman und anderen mehr einige der hervorragendsten Autoren des Landes vertreten sind.

Noch bedeutungsvoller war die Frage, wann der Verlag es wagen würde, auch in das elektronische Publizieren einzusteigen, denn hier hatte sich analog zur Entwicklung in Polen - längst der global agierende Konkurrent Wolters Kluwer mit seiner tschechischen Tochterfirma «Aspi» breitgemacht. Ein erster, noch recht schüchterner Schritt für Beck war die Übernahme der elektronischen Datenbank «LexData» im Jahre 20o2, die zumindest im Bereich der Gerichtsentscheidungen ein in etwa ebenbürtiges Angebot darstellte. Doch es war bald erkennbar, dass damit allein die jahrelange Dominanz von «Aspi» nicht geschwächt werden konnte. Hans Dieter Beck entschied sich wiederum für den Weg über die Kommentarliteratur. So gab es im Jahr 2008 einen elektronischen Neubeginn unter dem Namen «beck-online.cz» mit zunächst sieben thematischen Fachmodulen, bei denen die Präsenz der Beck'schen Autorenwerke ganz im Vordergrund stand. Dieser Schritt hat sich in der Tat als wirksam erwiesen, und das tschechische «beck-online» erfreut sich seitdem guten Wachstums.

Der bis dahin letzte Schritt auf verlegerisches Neuland erfolgte schließlich ab dem Jahre 2010. Mit zunächst nur zwei Mitarbeitern vor Ort und unter der organisatorischen Leitung des Prager Verlags wurde eine Niederlassung in Bratislava gegründet, um von dort aus auch im Nachbarland Slowakei die juristische Literatur zu bereichern. Diese Aktivität beschränkt sich bislang auf ein schmales juristisches Buchprogramm, das aber doch schon einige sichtbare Spuren in den Bibliotheken und Buchhandlungen des Landes hinterlassen hat.

Der Blick auf die heutige Marktlage in der Tschechischen Republik offenbart manche Parallele zu der eben bereits geschilderten Situation in Polen. Nicht zuletzt dank eines traditionsreichen Namens und bewährter verlegerischer Konzepte ist es gelungen, aus kleinen Anfängen eine landesweit anerkannte Qualitätsmarke zu entwickeln. Der Prager Beck Verlag erfreut sich eines außerordentlich hohen Ansehens in den Bereichen Bücher und Zeitschriften und hält dort einigen Abstand zu seinen Konkurrenten, während im elektronischen Bereich versucht wird aufzuholen. Darum bemühen sich derzeit knapp 4o Mitarbeiter in Prag und Bratislava, die 
neben fünf Zeitschriften und mehreren Online- und Offline-Produkten jährlich etwa 100 Buch- und Loseblatt-Titel produzieren.

\section{c) Der Verlag «All Beck» - Erwerb einer Beteiligung in Rumänien und später eine Art Neugründung}

Es liest sich wie die Story einer amerikanischen Tellerwäscher-Karriere: Bukarest im Frühjahr 1990, kurz nach der blutigen Revolution gegen das Ceauşescu-Regime. Fünf junge Männer, allesamt Lehrstuhlassistenten an verschiedenen Fakultäten, beschließen, etwas gegen den chronischen Mangel an Lehrbüchern zu unternehmen. Sie sammeln Geld von den betroffenen Studenten, händigen ihnen dafür Bezugsscheine aus, beschaffen das wertvolle Papier für eine der wenigen funktionierenden Druckereien und organisieren erstmals seit Jahren einen Nachdruck von drei der am meisten benötigten Titel. Man hatte klug kalkuliert, und so konnten nicht nur die Inhaber von Bezugsscheinen bedient werden, sondern es blieben einige Tausend Exemplare für den freien Verkauf. Als auch der erfolgreich verlaufen war, war genügend Geld übrig, um davon - diesmal schon ohne Bezugsscheine - weitere Nachdrucke zu produzieren, die wiederum reißenden Absatz fanden. So und nicht anders kam etwas in Gang, das sich bis ins Jahr 1998 zum größten privaten Verlag Rumäniens gemausert hatte, rund 200 Mitarbeiter beschäftigte und mehr als $35^{\circ}$ Buchtitel pro Jahr veröffentlichte. Und eben das war die Situation der «Verlagsgruppe All», als Hans Dieter Beck im Juli 1998 dort eine Beteiligung erwarb.

Dass zwei der drei Titel, mit denen alles begonnen hatte, ausgerechnet juristische Bücher waren, war zunächst nicht mehr als eine zufällige Begebenheit gewesen. Aber im Laufe der Zeit hatte sich dieser Trend fortgesetzt, und die «Verlagsgruppe All» hatte neben Schulbüchern und naturwissenschaftlichen Lehrbüchern vor allem auch juristische Titel in ihrem Programm. Die Konstellation, die C.H.Beck hier vorfand, war allerdings nicht frei von Tücken. Man erwarb eine $50 \%$ - Beteiligung an einer neu gegründeten Verlagsgesellschaft namens «All Beck AG», in die zuvor alle juristischen und wirtschaftswissenschaftlichen Titel überführt worden waren. Nicht genug damit, dass Beck also in dieser Firma nicht «das Sagen» hatte - nein, man war auch nur an einem von vier Verlagen innerhalb der Gruppe beteiligt, und dieser Verlag bestand im Grunde nur aus einem Lektorat und war in allen anderen Angelegenheiten vom Management und von den anderen Abteilungen der Gruppe abhängig. Diese Struktur bescherte dem Verlag All Beck erwartungsgemäß einige Probleme, die nach nur zweieinhalb Jahren zur Abspaltung von der restlichen Verlagsgruppe 
führen sollten. Gegen Ende des Jahres 2000 kam es zu tiefgreifenden Meinungsverschiedenheiten zwischen den rumänischen Firmengründern, und am 1. Januar 2001 zog einer dieser «fünf jungen Männer» mit der kleinen Mannschaft des juristischen Teilverlags aus dem Bürogebäude der Verlagsgruppe All aus.

Der Verlag All Beck startete somit unter neuen Vorzeichen in eine neue Existenz. In eigenen (mehr als bescheidenen) Büroräumen, aber in voller Souveränität bezüglich der bisher so umstrittenen Programm- und Vertriebspolitik vollzog sich nun auch in Bukarest eine Art Neugründung in dem ohnehin schwierigen, weil von Armut und niedriger Kaufkraft geprägten rumänischen Markt. Mit der neu gewonnenen Selbständigkeit konnte Beck nicht nur seine Beteiligungsquote auf $75 \%$ erhöhen, sondern - nach einer mühevollen Anlaufphase - auch verlegerisch mehr erreichen als noch zuvor.

Traditionell hatte das Verlagsprogramm zunächst fast nur aus akademischen Titeln, insbesondere Lehrbüchern, bestanden, während juristische Praktikerliteratur im ganzen Lande weitgehend unbekannt war. All Beck nutzte nun seine vergleichsweise starke Marktstellung und begann allmählich damit, Autoren und Kunden von der Notwendigkeit andersartiger Werke zu überzeugen. So entstanden neue Zeitschriften, allen voran der Entscheidungsdienst «Buletinul Casației» in Kooperation mit dem Obersten Gerichtshof, eine erste CD-ROM und natürlich auch diverse neue Buchreihen, wie z. B. die Textausgabenserie «La Zi» («Aktuell») und die Kommentarreihe «Comentarii All Beck». Nach und nach erschienen nun auch in Rumänien solide Kommentierungen zu den wichtigen Gesetzbüchern, so etwa zum Handelsgesetzbuch (herausgegeben von Cărpenaru), zur Zivilprozessordnung (Leş) und zu dem erst kürzlich neu in Kraft getretenen Bürgerlichen Gesetzbuch (Baias). Hinzu kamen erste praxisbezogene Hand- und Formularbücher, und so nahm schließlich auch das Verlagsprogramm in Bukarest, die typisch «Beck'schen» Konturen an. Im Jahr 2008 begann dann auch hier das elektronische Zeitalter. Unter der Marke «Legalis» startete eine juristische Online-Datenbank mit der vollständigen Gesetzgebung in konsolidierter Form (einschließlich der historischen Fassungen zu jedem gewünschten Stichtag), mit umfangreicher Rechtsprechung und mit einer kleinen Auswahl an Literatur.

Bei all dieser Entwicklung ist jedoch nicht zu übersehen, dass der rumänische Markt für das juristische Publizieren ein nach wie vor schwieriges Terrain darstellt. Kaufkraft und Kaufbereitschaft der juristischen Berufe befinden sich auch heute noch auf einem sehr niedrigen Niveau, während 
die politische wie auch die wirtschaftliche Entwicklung des Landes nur zögerlich vorankommen und immer wieder Rückschläge erleiden. Was dem Verlag aber bleibt, ist eine vielversprechende Ausgangsposition in einem Land mit rund 22 Millionen Einwohnern, die für die Zukunft hoffen lässt. Die Gegenwart ist freilich noch geprägt von einer sprunghaften Gesetzgebung, die nicht selten mittels «Eilverordnung» der Regierung und damit praktisch im Verborgenen stattfindet und dem Lektorat immer wieder neue Überraschungen bereitet. Insofern ist es nicht ganz leicht, in diesem Umfeld seriöse juristische Werke zu planen und gar erscheinen zu lassen. Aber irgendwie scheint der Verlag, der mit seinen 50 Mitarbeitern nahezu 200 Buchtitel pro Jahr publiziert, ein Rezept dafür gefunden zu haben.

\section{Erwerb von Nomos und anderen Verlagen}

Die Ursprünge des Nomos Verlags in Baden-Baden gehen zurück bis 1936 . Damals hat August Lutzeyer, ein Wirtschaftsfachmann, den Berliner Lutzeyer Verlag gegründet, im Wesentlichen für Wirtschaftsfragen, und bald danach die Niederlassung in Bad Oeynhausen bei Minden/Westfalen, in der auch juristische Literatur erschien. In den vierziger Jahren während und nach dem Ende des Zweiten Weltkriegs findet man den Verlag nicht nur in Berlin und Bad Oeynhausen, sondern auch in Frankfurt am Main, Leipzig, Minden/Westfalen und seit 1955 auch in Baden-Baden. 1963/64 erhielt er den Namen Nomos Verlagsgesellschaft und gehörte wohl schon zum Frankfurter Suhrkamp Verlag. Das Wort Nomos (griechisch: Gesetz) deutet daraufhin, dass nun das Recht im Vordergrund stand. Suhrkamp verkaufte ihn Ende 1998 an den Berliner wissenschaftlichen Springer Verlag, der kurz danach von Bertelsmann übernommen wurde. Bertelsmann wollte ihn loswerden, weil Springer als naturwissenschaftlich, technisch und medizinisch interessiertes Unternehmen für die Verwaltung des verhältnismäßig kleinen und schwierigen Verlags in Baden-Baden nicht genug Erfahrung hatte, denn der beschäftigte sich mit Veröffentlichungen im Bereich der Rechts-, Politik- und Sozialwissenschaften.

Deshalb bot man ihn C.H.Beck an. Die Verhandlungen fanden 1999 statt und waren schwierig, weil Bertelsmann einen sehr hohen Preis verlangte und die Abonnentenzahl des dreißigbändigen Loseblattwerks «Deutsches Bundesrecht» zurückging, mit dem der gesamte Nomos Verlag finanziert wurde. Schließlich gab Hans Dieter Beck nach, zog aber sein Angebot schnell wieder zurück, um dann nach heftigen Protesten und Drohungen 
aus Baden-Baden doch noch abzuschließen, auch in der Überlegung, dass Nomos in den Händen ehrgeiziger Konkurrenten dem Beck'schen Verlag schaden könnte, zumal dort in Baden-Baden sehr früh ein Schwerpunkt des Europarechts entstanden war. Der Konkurrent würde wohl Wolters Kluwer gewesen sein. Das war nun der letzte Wechsel in der Geschichte dieses eher kleinen Unternehmens.

Im Gegensatz zum Vahlen Verlag, der vollständig und auch räumlich in den Münchner Verlag eingegliedert worden ist, blieb Nomos als Teil der Gruppe C.H.Beck äußerlich selbständig. Das führte zu Schwierigkeiten in der Zusammenarbeit mit dem bisherigen Geschäftsführer. Nach drei Jahren wurde er abgefunden. Sein Nachfolger wurde Alfred Hoffmann, der bisher den Deutschen AnwaltVerlag in Bonn geleitet hatte. Nun ging es aufwärts. Die meisten der unvollständig gebliebenen Loseblattausgaben wurden als gebundene Werke herausgebracht, die Zeitschriften redaktionell besser organisiert, neue gegründet, Hand- und Formularbücher für Anwälte herausgegeben und mehrere Hand- und Großkommentare. Besonders der arbeits- und sozialrechtliche Teil ist vergrößert worden und die Abhängigkeit des Verlags von veralteten Loseblattwerken beseitigt. So hat er sich zu einem einfallsreichen Unternehmen auf neuen Wegen entwickelt. Der hohe Preis, den Hans Dieter Beck gezahlt hat, war gut angelegt.

In dieser Zeit hat Hans Dieter Beck noch zwei andere Verlage erworben, nämlich Helbing \& Lichtenhahn und den Kommunal- und Schul-Verlag. Helbing \& Lichtenhahn hat seinen Sitz im schweizerischen Basel. Die Schweiz hat 7 Millionen Einwohner und 7000 Anwälte, Deutschland 80 Millionen Einwohner und inzwischen 160 ooo Rechtsanwälte. Deutschland hat eine Amtssprache, nämlich deutsch, die Schweiz drei, nämlich deutsch, französisch und italienisch. Deutschland hat eine Zivilprozessordnung, die Schweiz hatte damals - vor der ZPO-Reform des Jahres 2008 - noch 27 , nämlich je eine der 26 Kantone und die des Bundesgerichts. Der Verlag ist fast einhundert Jahre alt und einer von dreien, die Literatur zum Schweizer Recht veröffentlichen. 1998 wurde er Hans Dieter Beck zum Kauf angeboten, der ihn erworben hat mit einigen Anfangsschwierigkeiten, wie man sich denken kann. 2003 übernahm der Schweizer Jurist und Verleger Men Haupt die Leitung dieses für die Schweiz wichtigen juristischen Verlags, dessen etwas mehr als zwanzig Mitarbeiter jährlich 80 neue Buchtitel und sechs Zeitschriften veröffentlichen, außerdem auf der technischen Plattform von beck-online eine Datenbank betreiben, die weiter ausgebaut wird. 
Der Kommunal- und Schul-Verlag in Walluf bei Wiesbaden wurde 2003/2004, von Hans Dieter Beck gekauft. Es ist ein kleiner Betrieb mit fünfzehn Mitarbeitern, die auf 400 ooo Seiten das gesamte Recht für deutsche Gemeinde-, Stadt- und Kreisverwaltungen in sieben westlichen und fünf östlichen Bundesländern in je 30 beziehungsweise 24 . Loseblattordnern veröffentlichen. Knapp die Hälfte des Inhalts besteht aus jeweils 100 Kommentaren oder Abhandlungen zum Landesrecht, die andere aus ungefähr 100 Beiträgen zum Bundesrecht. Der Aufwand für die Einordnung ist ziemlich groß. Deshalb nimmt die Zahl der Abonnenten langsam ab. Aber man hat einen Ausweg gefunden und die «Praxis der Kommunalverwaltung» - so heißt das Hauptwerk - inzwischen digitalisiert und nun kann es über beck-online benutzt werden, so dass der kleine Verlag wieder eine sichere Zukunft hat.

\section{4. «Beck International» - das englischsprachige Programm des Verlages}

Der Markt für juristische Publikationen in deutscher Sprache ist von wenigen Ausnahmen abgesehen auf den deutschen Sprachraum begrenzt. Darüber hinaus ist ein nennenswerter Absatz, vor allem bei Praktikerwerken, kaum zu erzielen. Englisch als international akzeptierte Publikationssprache dagegen ermöglicht es dem Verlag, diese Sprachbarriere zu überwinden. Englisch ist lingua franca der Wirtschaft und immer mehr auch des Rechts. Im Zuge der Globalisierung von Wirtschaft und Recht nutzen nicht muttersprachlich englische oder amerikanische Juristen immer häufiger Englisch als die Sprache der Wahl. Dies gilt gleichermaßen für Publikationen, die nur in englischer Sprache europaweit und international zur Kenntnis genommen und zitiert werden. Dafür schafft «Beck International» eine Publikationsplattform, nicht zuletzt auch im Interesse seiner Autoren.

Die deutsche Rechtstradition unterscheidet sich von der englischen und US-amerikanischen common-law-Tradition nicht unerheblich. Während sich die letztere aus der Auslegung praktischer Fälle entwickelt, wird die Rechtstradition der deutschsprachigen Länder durch ihre Wissenschaftlichkeit, ihre terminologische Klarheit und nicht zuletzt auch ihren rechtsdogmatischen Ansatz gekennzeichnet. Damit füllt die deutsche Rechtstradition auch publizistisch Lücken.

Angesichts dieser Situation musste der Verlag Überlegungen anstellen, seinen Markt über die bestehenden Sprachgrenzen hinaus auszuweiten. 
Da auch deutsche Autoren interessiert sind, im Ausland wahrgenommen zu werden, war es sinnvoll, ihnen im Rahmen des Verlagsprogramms eine zusätzliche Publikationsmöglichkeit zu schaffen. Und schließlich: Es besteht ein elementares Interesse, eine internationale Plattform für Publikationen zur deutschen Rechtssituation zur Verfügung zu stellen. Nur so kann deutsches Recht insbesondere in Staaten wirksam werden, die noch damit befasst sind, ihre eigene Rechtsordnung aufzubauen.

Anfang der 1990er Jahre wurden infolge solcher Überlegungen die ersten englischsprachigen Titel in das rechtswissenschaftliche Programm aufgenommen. Am Anfang stand das von Bernd Rüster herausgegebene vierbändiges Loseblattwerk «Business Transactions in Germany», gefolgt von einem von den Professoren Bruno Simma, Hermann Mosler, Rüdiger Wolfrum, Christian Tomuschat und Albrecht Randelzhofer herausgegebenen Kommentar zur «Charter of the United Nations». In den Jahren danach folgten unter der Reihenbezeichnung «German Law Accessible» etwa ein Dutzend Titel, die sich vor allem mit Themen des deutschen Wirtschaftsrechts befassten.

2008 wurde beschlossen, das Programm systematisch auszubauen. Während bislang im Wesentlichen deutsches Recht thematisiert wurde, erschließt das unter dem Titel «Beck International» zusammengefasste Programm nunmehr alle Gebiete des europäischen, ausländischen und internationalen Rechts, und zwar sowohl in der Form systematischer, nach Lebenssachverhalten geordneter Handbücher wie auch als klassische «Artikel-für-Artikel»- Kommentare.

«Beck International» basiert von Anfang an auf einer festen Kooperation mit dem Oxforder Verlag Hart Publishing und dem Beck'schen Tochterverlag Nomos. Der Helbing \& Lichtenhahn Verlag aus Basel kooperiert von Fall zu Fall. Die Verlage treten unter dem Namen «C.H.Beck - Hart Nomos» gemeinsam auf; jeder Partner ist berechtigt, Werke in die Kooperation einzubringen. Hart hat sich dabei nicht nur als geeigneter Ko-Verlag, sondern, dank seiner beträchtlichen Reputation und seines guten Netzwerks in Großbritannien und den USA auch als vorzüglicher Vertriebspartner erwiesen.

2013 umfasst das Programm von Beck International bereits mehr als 5o Titel vor allem aus den Bereichen Arbitration/ADR/Litigation, European and International Criminal Law, European and International IP Law, European Union Law und Public International Law. Beispielhaft genannt werden soll der bereits in mehreren Auflagen erschienene «Triffterer, Commentary on the Rome Statute on the International Criminal Court», 
«Wolff, The New York Convention Commentary», «Schütze, Institutional Arbitration - Commentary» und der von Bertrand Waegenbaur verfasste «EU Court of Justice - Commentary on Statute and Rules of Procedure».

Der klassische, den Bestimmungen einer Norm folgende Kommentar ist für die deutschsprachige Rechtsliteratur kennzeichnend. Er gehört zum täglichen Handwerkszeug eines jeden Juristen in Deutschland, Österreich und der Schweiz. Anders ist die Lage im Ausland, insbesondere in den common law Ländern: Ausgehend vom prakischen Fall stehen dort im Mittelpunkt Gesetzessammlungen und Praxishandbücher; Kommentare waren zumindest in der Vergangenheit sehr selten oder entsprachen nicht dem Anspruch, der an einen wissenschaftlich fundierten Kommentar zu stellen gewesen wäre. Um sich vom internationalen Wettbewerb abzusetzen und sein spezielles Verlags-Know-How zu nutzen, setzt «C.H.Beck Hart - Nomos» bei seiner Programmentwicklung auf das spezielle Format des Kommentars. Damit entspricht er der Nachfrage nach article-by-article commentaries, die mit der Zunahme kodifizierten Rechts spürbar wächst. Auf diese Weise bekennt sich «C.H.Beck - Hart - Nomos» zu einer Dogmatisierung des Rechts, d. h. zu einer aus einer Norm deduktiv abgeleiteten wissenschaftlichen Deutung der einzelnen Rechtssätze. Die Auswahl der Autoren auch aus anderen Rechtstraditionen gewährleistet dabei die nötige Distanz und Ausgewogenheit.

Beim Aufbau des Verlagsprogramms erweist sich die Publikationssprache Englisch naturgemäß als Hürde. Grundsätzlich wird vom Autor ein genuin englischsprachiges Manuskript erwartet, wobei sich die Ansprüche an die Qualität der Sprache im Laufe der Zeit relativierten. Das von einem nicht muttersprachlich englischen Autor verfasste Werk wird in aller Regel nicht klingen wie das eines Briten oder US-Amerikaners. In der Tat kann es so nicht klingen, da es von einem etwa in der deutschen, italienischen oder polnischen Rechtstradition geschulten Verfasser erdacht und formuliert wurde, dessen juristische Argumentation anders verläuft als die seiner englischen oder amerikanischen peer group. Der von «C.H.Beck - Hart - Nomos» gestellte sprachliche Anspruch richtet sich daher auf den korrekten Gebrauch der Fachterminologie, des Fachjargons und der Regeln der Grammatik und der Syntax. Es wird hingenommen, dass sich dieser «technische» Sprachgebrauch deutlich vom literarischen Englisch unterscheidet. Dies fällt umso leichter, als die Mehrheit der Leser - wie die Autoren auch - Englisch als lingua franca nutzen und mit einer «technischen» Sprache häufig besser zurechtkommen als mit dem Englisch eines Muttersprachlers. Ein nützlicher Maßstab für Englisch als 
Publikationssprache haben sich dabei die Sprachratgeber der Europäischen Kommission und der Sprachgebrauch der europäischen Institutionen, insbesondere des Gerichtshofes, erwiesen.

Die größte Herausforderung für einen internationalen Verlag ist, einen weltweiten Vertrieb der englischsprachigen Werke zu gewährleisten. In der Vergangenheit erfolgte dies über projektbezogene Zusammenarbeit (z. B. mit Matthew Bender und Oxford University Press) und institutionalisierte Partnerschaften. Eine solche institutionalisierte Partnerschaft ist Law Publishers in Europe (LPE), eine Vereinigung von 12 großen Rechtsverlagen aus verschiedenen Mitgliedstaaten der Europäischen Union, die werkbezogen Vertriebskooperationen bilden.

\section{Neue oder grundlegend veränderte Rechtsgebiete}

Nicht nur politische Veränderungen brachten nach 1990 neue Herausforderungen für den Verlag C.H.Beck. Vielmehr galt es auch, auf neue Rechtsgebiete oder auf wesentlich veränderte Rechtsfragen zu reagieren. Dafür hier nun einige Beispiele:

\section{a) Informationstechnologie, Telekommunikation, Datenschutz}

Mit der Erfindung serienmäßig produzierter Mikroprozessoren in den 1970er Jahren, die bereits die dritte Generation der Computerentwicklung markiert, wurden Computer zunehmend leistungsfähiger, kleiner und kostengünstiger. Dennoch wurden zunächst deren Möglichkeiten verkannt. In Haushalte zogen Computer daher erst in den 1980er Jahren ein, zunächst als 8 Bit-Mikroprozessoren und mit noch recht beschränkten Arbeitsspeichern, dann aber zunehmend als immer leistungsfähigere Geräte. In den 199oer Jahren kamen das Internet und das World Wide Web dazu. Spätestens zu Beginn des 21. Jahrhunderts sind Computer im beruflichen und privaten Verkehr omnipräsent. In digitalen Rechnern werden Elemente der Bildbearbeitung und der Telekommunikation integriert. All dies sind technische Entwicklungen, an denen auch das Recht nicht vorbeigehen konnte. Gesetzliche Regelungen, die sich ausschließlich oder auch mit der neuen digitalen Welt befassen, werden inzwischen unter dem Schlagwort «IT-Recht» behandelt. Daneben hat das Telekommunikationsrecht 1996 das alte Fernmelderecht abgelöst. Mit dem Wegfall des staatlichen Fernmeldemonopols zählt auch die Regulierung der Telekommunikationsnetze zu seinen wesentlichen Materien. Sie soll allen Wettbe- 
werbern einen diskriminierungsfreien Netzzugang dazu ermöglichen. Die zunehmende Digitalisierung von Informationen hat schließlich das Datenschutzrecht aus seinem «Dornröschenschlaf» erweckt.

IT-Recht ist die Kurzform für Informationstechnologierecht. Es ist nicht klar definiert, umfasst aber - mit unterschiedlichen Schwerpunkten mehrere andere Teilrechtsgebiete. Dazu zählen: Internetrecht, Multimediarecht, Recht der elektronischen Medien, Telemedienrecht, aber auch die noch aus den 1980er- und 199oer-Jahren stammenden Begriffe Computerrecht und EDV-Recht. Einen Versuch der Eingrenzung unternimmt die Fachanwaltsordnung, in der 2006 mit der Einführung des Fachanwalts für Informationstechnologierecht die relevanten Gebiete beschrieben werden: u.a. Vertragsrecht, Recht des elektronischen Geschäftsverkehrs, Immaterialgüterrecht mit den Bezügen zum Kennzeichen- und Domainrecht, Datenschutz- und Datensicherheitsrecht, Telekommunikationsrecht, eGovernment, Internationales Privatrecht, Straf- und Prozessrecht, soweit diese Besonderheiten im Bereich der Informationstechnologien

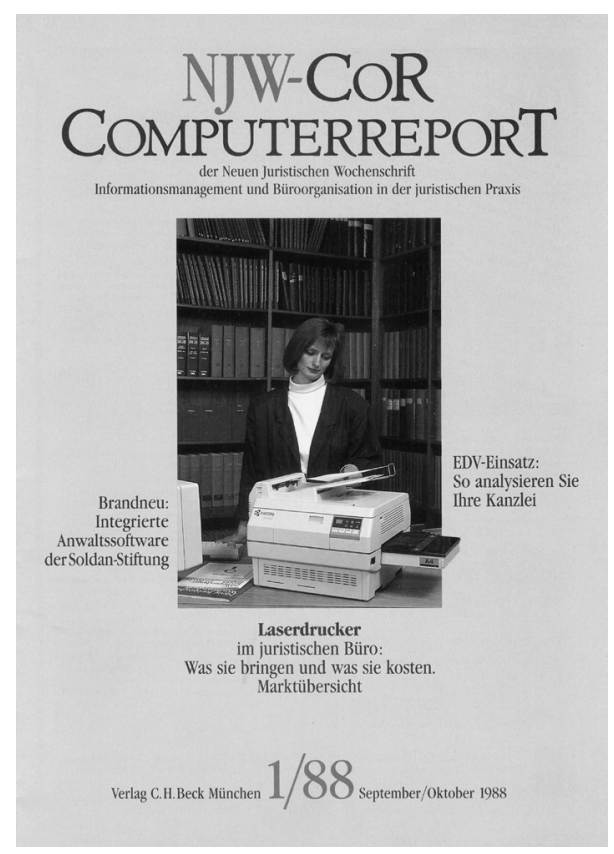

Das erste Heft von NJW-CoR aufweisen. Teilweise als eigenständige Rechtsgebiete werden dagegen das Telekommunikationsrecht und das Datenschutzrecht gesehen. Überschneidungen gibt es ebenfalls mit dem Rundfunkrecht, das im Übrigen als eigene Rechtsmaterie eingeordnet werden dürfte.

Der Verlag hat sich dem Thema vorsichtig genähert. Pionier war ab 1988 der «NJW-Computerreport (NJW-CoR)», der der NJW erst sechs, dann acht Mal pro Jahr beigelegt wurde. Rechtsfragen spielten dort nur eine untergeordnete Rolle, es ging vor allem um die Fragen der praktischen Anwendung der elektronischen Datenverarbeitung im juristischen Bereich. Ziel der Zeitschrift war es, den Juristen mit wichtigen Informationen über neue Geräte und Programme $\mathrm{zu}$ versorgen und so die damals noch ausgeprägten Berührungsängste gegenüber der modernen Technik abzubauen. Der Leser sollte in die Lage versetzt werden, vernünftig über den Erwerb der richtigen Produkte (nicht zuletzt auch des Verlages C.H.Beck) zu entscheiden, ihre Bedienung zu be- 
herrschen und sein Büro sachgerecht zu organisieren. Dazu erschienen Rezensionen und Vergleichstests zu Hardware, Software und Datenbanken wie etwa zu den familienrechtlichen Berechnungen von Gutdeutsch, juris oder zur NJW-Leitsatzkartei auf CD-ROM. Ganz ungewöhnlich für eine Zeitschrift im juristischen Programm waren die Verwendung von zahlreichen Abbildungen, einem stets wechselnden Umschlagbild und die Streuung von Anzeigen im ganzen Heft. Mit steigenden Anzeigenerlösen konnte das Blatt eine Zeit lang an Volumen zulegen. 2001 wurde NJW-CoR allerdings nicht mehr weiter produziert. Der Computerreport hatte seinen Auftrag zur Einführung in die digitale Welt erfüllt.

1998, also zehn Jahre später, erschien dann erstmals die Zeitschrift «MultiMedia und Recht - Zeitschrift für Informations-, Telekommunikations- und Multimediarecht (MMR)». Sie informiert seitdem umfassend über alle Bereiche des Informations-, Telekommunikations- und Medienrechts. Berichtet wird über aktuelle Rechtsprechung, die nationale und europäische Gesetzgebung, die technologischen Entwicklungen mit ihren juristischen und wirtschaftlichen Folgen, die Aktivitäten der europäischen und internationalen Institutionen wie auch über die Arbeit formeller und informeller Koordinations- und Gesprächskreise.

Der erste Meilenstein eines Werkes zum IT-Recht in Loseblattform hat noch etwas länger auf sich warten lassen. Er war dann 1990 das von Wolfgang Kilian, Professor an der Leibniz Universität Hannover und dort Gründer des Instituts für Rechtsinformatik, und Benno Heussen, Rechtsanwalt in Berlin und Honorarprofessor an der Universität Hannover, herausgegebene «Computerrechts-Handbuch». Es befasst sich mit allen Regelungen, die sich auf die rechtlichen Voraussetzungen und Folgen der Informationstechnik in den verschiedenen Lebensbereichen beziehen. Der Schwerpunkt der Darstellung liegt in der Analyse und Beschreibung der bestehenden rechtlichen Rahmenordnung und ihrer praktischen Problemfelder. Im Laufe der Jahre wurde es beständig den neuen Entwicklungen im Bereich des Computerrechts angepasst.

Bereits 1991 erschien das Werk «Softwareüberlassungsverträge» von Jochen Marly, damals noch Rechtsanwalt und Lehrbeauftragter an der Hessischen Verwaltungs- und Wirtschaftsakademie, inzwischen Professor für Zivilrecht, Gewerblichen Rechtsschutz und Urheberrecht sowie Recht der Informationsgesellschaft an der TU Darmstadt. Das Praxishandbuch hat sich schon mit der ersten Auflage als Standardwerk etabliert. Es stellt die heute gebräuchlichen Verträge über Erwerb und Einsatz von Software dar und beurteilt diese. Dabei werden auch die urheberrechtlichen Vor- 
schriften über den Rechtsschutz von Computerprogrammen sowie die Vorschriften über die Allgemeinen Geschäftsbedingungen erläutert. Inzwischen liegt das Handbuch in der 5. Auflage und unter dem Titel «Praxishandbuch Softwarerecht» vor. Dem Autor gelingt es seit über 20 Jahren, ohne sich als Herausgeber auf zahlreiche Verfasser zu stützen, als einziger Autor das Handbuch fortzuschreiben, dem schon in der Besprechung zur 1. Auflage das Prädikat «umfassend, praxisgerecht und niveauvoll» verliehen wurde (Hoeren, NJW 1992, 1745).

Das Spektrum der Literatur zum Multimediarecht wurde im Jahr 1999 um zwei weitere wichtige Loseblattwerke erweitert. Zunächst erschien das «Handbuch Multimedia-Recht». Der eine Herausgeber, Thomas Hoeren, war dem Verlag seit Veröffentlichung seiner Dissertation «Softwareüberlassung als Sachkauf» in der Praktikerreihe «Aktuelles Recht» im Jahre 1989 verbunden; das war schon doppelt ungewöhnlich. Inzwischen ist er Professor an der Westfälischen Wilhelms-Universität in Münster und Leiter der zivilrechtlichen Abteilung des Instituts für Informations-, Telekommunikations- und Medienrecht (ITM) geworden. Der zweite Herausgeber ist Ulrich Sieber, Direktor des Max-Planck-Instituts für ausländisches und internationales Strafrecht, Freiburg, damals noch Professor am Lehrstuhl für Strafrecht, Strafprozeßrecht und Informationsrecht der Universität Bayreuth. Der Kreis der Herausgeber wurde 2012 erweitert. Neu hinzugekommen ist Bernd Holznagel, Kollege von Hoeren in Münster, dort am ITM Leiter der öffentlich-rechtlichen Abteilung.

Das Handbuch, an dem neben den Herausgebern rund 6o namhafte Wissenschaftler und erfahrene Praktiker mitwirken, hat sich von Anfang an als Standwerk etabliert. Der schnelle Aufstieg des elektronischen Geschäftsverkehrs brachte eine Fülle von Rechtsfragen mit sich, die das Handbuch praxisorientiert behandelt. Der Aufbau des Werkes orientiert sich an den Fragestellungen und Abläufen im Unternehmen. Beginnend bei den technischen und wirtschaftlichen Grundlagen über die unzähligen Rechtsfragen im Online-Bereich, etwa dem Schutz von Kennzeichen oder von Texten, Bildern, Musik gegen rechtswidrige Verbreitung im Internet, die Möglichkeiten des Vertragsschlusses oder des Wertpapierhandels im Internet u.v. m., bis hin zur Haftung gibt das Werk für alle Schlüsselsituationen die notwendigen Erklärungen und Antworten. Die aktuelle Gesetzgebung und die Rechtsprechung werden laufend eingearbeitet. Inzwischen liegt bereits die im Juli 2013 erschienene 34. Ergänzungslieferung vor.

Nur wenige Monate nach dem Handbuch Multimedia-Recht erschien, ebenfalls als Loseblattwerk, der Kommentar «Recht der Multimedia- 
Dienste», herausgegeben von Alexander Roßnagel, Professor an der Universität Kassel und dort Direktor des Forschungszentrums für Informationstechnik-Gestaltung (ITeG). Das Werk ist praxisorientiert, aber wissenschaftlich fundiert und erläutert die durch das Informations- und Kommunikationsdienstegesetz (IuKDG) erlassenen Vorschriften (Teledienstegesetz, Teledienstedatenschutzgesetz, Signaturgesetz und Signaturverordnung). 2013 wurde das Buch konzeptionell überarbeitet und unter dem Titel «Beck'scher Kommentar zum Recht der Telemediendienste» in ein gebundenes Werk überführt.

In der «Grauen Reihe» erschien im Januar 2008 und Anfang 2011 in 2. Auflage der Kommentar «Recht der elektronischen Medien», herausgegeben von Gerald Spindler, Professor für Bürgerliches Recht, Handels- und Wirtschaftsrecht, Rechtsvergleichung, Multimedia- und Telekommunikationsrecht an der Universität Göttingen, und Fabian Schuster, Rechtsanwalt und Honorarprofessor an der Universität zu Köln. Der Kommentar erfasst die Kernmaterien des Medien- und Kommunikationsrechts und ist von dem Gedanken getragen, dass die Konvergenz der Medien langfristig auch zu einer Verschmelzung der Rechtsmaterien führen wird. Die zunehmenden, medienübergreifenden Rechtsfragen werden in diesem Kommentar deshalb auch integrativ behandelt, ein - im Vergleich zu sonstigen Informationsquellen - «unschlagbares und herausragendes Alleinstellungsmerkmal» (so Lindhorst, MMR 6/2009, XXIX).

Auch im Bereich des Rundfunkrechts konnte mit dem 2002 erstmals und 2012 in der 3. Auflage erschienenen «Beck'schen Kommentar zum Rundfunkrecht» eine Lücke des Verlagsprogramms geschlossen werden. Die Herausgeber Werner Hahn, Justitiar des NDR, und Thomas Vesting, Professor für Öffentliches Recht, Recht und Theorie der Medien an der Universität Frankfurt am Main, haben zusammen mit Vertretern aus Wissenschaft und Praxis ein Werk geschaffen, das den Bedürfnissen einerseits nach Systematisierung, andererseits nach Hinweisen zur Lösung praktischer Fälle gerecht wird und gleichzeitig die Entwicklungen bei der Umgestaltung der Medienordnung berücksichtigt.

Die Bedeutung des Telekommunikationsrechts ist aufgrund der raschen technischen und gesellschaftlichen Entwicklungen im Bereich von Telekommunikation und Multimedia nicht zu unterschätzen. Durch die Postreform II 1994, wurde der Weg frei gemacht für die vollständige Liberalisierung des Telekommunikationsbereichs. Gleichzeitig waren die Mitgliedstaaten seitens der EU aufgefordert, bis Anfang 1998 die Netz- und Telefondienstmonopole aufzuheben. Dem war der Gesetzge- 
ber mit dem Telekommunikationsgesetz aus dem Jahr 1996 nachgekommen.

Der erste umfangreiche Kommentar, der «Beck'sche Kommentar zum TKG» erschien bereits Ende 1997 und hat sich umgehend eine herausragende Position in der Literatur zu diesem Rechtsgebiet gesichert. Herausgegeben wurde er von einem Kreis von im Telekommunikationsrecht erfahrenen Praktikern, den Rechtsanwälten Wolfgang Büchner, Jörg Ehmer, Martin Geppert, Bärbel Kerkhoff, Hermann-Josef Piepenbrock, Raimund Schütz und Fabian Schuster. Der Kreis der Herausgeber und Autoren hat sich von Auflage zu Auflage geändert, im Juni 2013 erschien die 4. Auflage des Kommentars, nunmehr herausgegeben von den Rechtsanwälten Dr. Martin Geppert und Dr. Raimund Schütz. Der Kommentar wird in der Praxis nicht zuletzt wegen der durchgängigen Berücksichtigung europarechtlicher Bezüge besonders geschätzt. - In der Reihe der «Grauen» Kommentare wurde daneben einweiteres Werk zu diesem Rechtsgebiet veröffentlicht. Mit Klaus-Dieter Scheurle, dem ersten Präsidenten der Regulierungsbehörde für Telekommunikation und Post (seit 2005 Bundesnetzagentur), und Rechtsanwalt Thomas Mayen, inzwischen auch Honorarprofessor an der Universität zu Köln, konnten zwei kompetente Herausgeber gewonnen werden.

Das Datenschutzrecht wurde zwar schon 1970 mit dem Hessischen Datenschutzgesetz und dann 1977 mit dem Bundesdatenschutzgesetz begründet. In den Fokus der juristischen und politischen Aufmerksamkeit rückte es erst das Bundesverfassungsgericht mit dem im Volkszählungsurteil 1983 (BVerfGE 65, 1) aus Art. 2 Absatz 1 des Grundgesetzes abgeleiteten «Recht auf informationelle Selbstbestimmung». Trotzdem fristete der Datenschutz noch über Jahre hinweg ein Schattendasein. Erst die zunehmende Digitalisierung und Vernetzung mit ihren Möglichkeiten, Daten zu sammeln und auszuwerten, ließ den Datenschutz zu einem wichtigen, zunehmend präsenten Diskussionsthema werden.

Schon früh hat der Verlag einen ersten Kommentar zum Bundesdatenschutzgesetz in der Gelben Reihe veröffentlicht. Das von Ministerialdirektor Hans-Joachim Ordemann und Regierungsdirektor Rudolf Schomerus verfasste Erläuterungsbuch konnte sich schon bald nach seinem Erscheinen 1977 als Standardwerk etablieren. Ab der 5.Auflage, 1992, löste Peter Gola, Professor an der Verwaltungsfachhochschule in Wiesbaden, Hans-Joachim Ordemann als Autor ab. Nach dem Tod von Rudolf Schomerus wird der «Gola/Schomerus» seit der 10. Auflage, 2010, bearbeitet von Peter Gola, Rechtsanwalt Christoph Klug und Oberverwaltungs- 
rätin Barbara Körffer. - Mit dem von Alexander Roßnagel herausgegeben «Handbuch Datenschutzrecht», erschienen 2003, ist es diesem zusammen mit rund 6o erfahrenen Praktikern und Wissenschaftlern gelungen, die Rechtsprobleme des Datenschutzrechts wissenschaftlich aufzuarbeiten und der Rechtspraxis, aber auch der Rechtspolitik, Orientierung und Sicherheit in einem unübersichtlichen Handlungsfeld zu bieten.

Der heutige Bundesbeauftragte für den Datenschutz und die Informationsfreiheit, Peter Schaar, verfasste bereits im Jahr 2002 bei C.H.Beck die wegweisende Monographie «Datenschutz im Internet», die einen Überblick über die datenschutzrechtlichen Vorgaben für das Internet gab.

Das Thema Datenschutz war auch Anlass für eine weitere Zeitschriftenneugründung im Verlag. Die «ZD - Zeitschrift für Datenschutz» startete im September 2011 und hat es sich zur Aufgabe gemacht, als Forum den Austausch und die Diskussion aktueller und grundlegender datenschutzrechtlicher Themen sowie damit zusammenhängender Aspekte zu bieten und dem Anwender den Zugang zu praxisorientierten Lösungen zu eröffnen.

\section{b) Energierecht}

Das Energierecht ist nicht neu. Sein zentrales Gesetz, das Energiewirtschaftsgesetz, ist schon am 13. Dezember 1935 erlassen worden. Es hat die damals bestehende wirtschaftliche Praxis der Energieversorgung im wesentlichen festgeschrieben. Danach gab es vorwiegend Stadtwerke, die zuständig für die Energieversorgung waren. Ihre Gebietsmonopole sicherten Konzessions- und Demarkationsverträge. Die Beibehaltung der deshalb vornehmlich dezentralen Energieversorgung hatte für den NS-Staat aber auch politische Gründe: Bei einer zentralen Energieproduktion hätten gezielte Luftangriffe schnell die Energieversorgung im Deutschen Reich lahmlegen können.

Selbst wenn das Energiewirtschaftsgesetz damit auch politischen Zielen der Kriegswirtschaft diente, hat es die Jahrzehnte der Bonner Republik weitgehend unbeschadet überstanden. Dann aber gab es einschneidende Veränderungen. Die erste steht im Zeichen von Privatisierung und Wettbewerb und wurde von der Europäischen Union angestoßen, die sich nicht zuletzt nach US-amerikanischem Vorbild - zum Ziel gesetzt hat, die gesamte staatlich dominierte Grundversorgung in die Hände einer wettbewerbsorientierten Privatwirtschaft zu legen. Auslöser für die zweite Änderung, die «Energiewende», war die Atomkatastrophe im japanischen Fukushima im März 2011. Dies führte bereits im Juni 2011 zum Beschluss 
der Bundesregierung, aus der Atomenergie auszusteigen. Der Anteil der erneuerbaren Energien (insbesondere Wind- und Solarenergie sowie Energie aus Biomasse) an der Stromversorgung soll dagegen bis 2020 auf $35 \%$, bis 2050 gar auf $80 \%$ steigen.

Die Vorgabe für die privatisierte, wettbewerbsorientierte Energieversorgung ist die «Richtlinie 96/92/EG betreffend gemeinsame Vorschriften für den Elektrizitätsbinnenmarkt aus dem Jahre 1996. Seit ihrer Umsetzung im Energiewirtschaftsgesetz 1998 haben Stromnetzbetreiber dritten Stromanbietern einen diskriminierungsfreien Netzgang zu gewähren. Seit 2005 wird der Netzzugang reguliert. Dazu werden Netzbetreiber von Regulierungsbehörden überwacht. Das ist nun die Bundesnetzagentur mit Sitz in Bonn. Die «Energiewende» hat die Förderung erneuerbarer Energie und das dazu erlassene Gesetz weit in den Vordergrund gerückt. Der schnelle Umstieg auf regenerative Energien löst aber auch zahlreiche logistische Probleme mit weitreichenden wirtschaftlichen Folgen aus. Insbesondere die Windenergie lässt sich vorwiegend nur in Norddeutschland und nicht zu vorherbestimmten Zeiten gewinnen. Das führt einerseits zu temporären Überlastungen der Stromnetze, andererseits wird der Strom nicht mehr dezentral am Ort der Energieversorgung erzeugt. Unerlässlich ist deshalb der unverzügliche Ausbau der Stromnetze, der den Stromtransport von Nord nach Süd ermöglicht. Dazu gibt es nun das Netzausbaubeschleunigungsgesetz.

Liberalisierung des Energiemarkts und «Energiewende» haben nicht nur zu einem enormen technischen, wirtschaftlichen und logistischen Umbau der Energieversorgung geführt. Sie haben auch eine geradezu explosionsartige Zunahme energierechtlicher Vorschriften bewirkt. Kam das Energiewirtschaftsgesetz von 1935 noch mit zwanzig zumeist kurzen Paragraphen aus, hat sich deren Zahl inzwischen versiebenfacht. Damit nicht genug: Längst ist das Energiewirtschaftsgesetz in eine Vielzahl von energierechtlichen Gesetzen und Verordnungen eingebettet, die problemlos mehrere Tausend Seiten füllen.

Die gesamte Entwicklung des Energierechts hat im Verlag allein das heute von Wolfgang Danner, vormals Präsident des Bundesamtes für Wirtschaft und Ausfuhrkontrolle und nunmehr Rechtsanwalt in Berlin, und Christian Theobald, Rechtsanwalt und Honorarprofessor an der Deutschen Universität für Verwaltungswissenschaften in Speyer, herausgegebene Loseblattwerk «Energierecht» begleitet. Es besteht bereits seit 1937, ist also nur zwei Jahre nach dem Erlass des Energiewirtschaftsgesetzes erschienen. Damals war es von Ernst Eiser begründet worden und 
erschien noch unter dem Titel «Energiewirtschaftsrecht» als gebundener Kommentar. Ab 1961 hatte die energierechtliche Entwicklung immerhin schon so zugenommen, dass das Werk als Loseblattwerk publiziert wurde. Lange blieb es dennoch bei einem Band. Wie das Energierecht, selbst ist inzwischen aber auch das Kommentarwerk explosionsartig angewachsen. Es besteht jetzt aus fünf Loseblattordnern mit rund 12 ooo Seiten. Nach Ernst Eiser folgten als Herausgeber: Johann Riederer, Frank Sieder und Wolfgang Obernolte. Letzterer war als Ministerialdirigent langjähriger Leiter der Energierechtsabteilung im Bundeswirtschaftsministerium und dort hat er Wolfgang Danner für das Werk gewinnen können, der bis 1992 ebenfalls im Ministerium tätig war.

Der «Danner/Theobald» erläutert die wesentlichen Rechtsgrundlagen zum Energiewirtschaftsrecht einschließlich des Regulierungsrechts, zum Recht der erneuerbaren Energien, zur Energieeinsparung, zum Energiekartellrecht, zum Energiesteuerrecht und zu diversen anderen energierechtlichen Bereichen. Kernstück des Werkes ist aber die Kommentierung des Energiewirtschaftsgesetzes geblieben, die inzwischen auf über zooo Seiten angewachsen ist. Aber nicht nur die im Energierecht zu verarbeitende Regelungsfülle, sondern auch die ständig kürzer werdenden Intervalle grundlegender Reformgesetze eines zumeist aus Brüssel getriebenen Bundesgesetzgebers machen es immer weniger möglich, zeitnah eine umfassende und aktuelle Erläuterung der maßgeblichen Vorschriften zu liefern. So mag sich der «Danner/Theobald» vielleicht schon bald zu einem Werk entwickeln, das eine deutlich abnehmende Kraft der Rechtsordnung dokumentiert, weil sie so von den Rechtsunterworfenen nicht mehr beherrscht und damit beachtet werden kann.

Bevor Christian Theobald Mitherausgeber und einer der maßggeblichen Autoren im «Danner/Theobald» wurde, hatte er sich bereits zu dem Verlagsautor entwickelt, der weit mehr als andere an energierechtlichen Werken und Publikationen beteiligt ist. Und diese Entwicklung hat sich fortgesetzt: Er ist Schriftleiter der seit 2004, erscheinenden Monatsschrift «Infrastrukturrecht - IR» und seit Ende 2012, tatkräftig unterstützt von Rechtsanwalt Peter Gussone, der «Zeitschrift für das gesamte Recht der Energiewirtschaft - EnWZ». Mit Jens-Peter Schneider, inzwischen Professor an der Universität Freiburg, gibt er das große Praxishandbuch «Recht der Energiewirtschaft» heraus, das demnächst bereits in 4. Auflage vorliegt. Gemeinsam mit seiner Ehefrau Christiane Nill-Theobald hat er die lehrbuchartige Darstellung «Einführung in das Energierecht» in mehreren Auflagen vorgelegt. Über zwei Auflagen hinweg war er Mitheraus- 
geber eines Kommentars zum Erneuerbaren-Energiengesetz. Gemeinsam mit Gabriele Britz, Professorin in Giessen, inzwischen Richterin des Bundesverfassungsgerichts, und Rechtsanwalt Christian Held gibt er die im Verlag erscheinende Schriftenreihe «Energie- und Infrastrukturrecht» heraus. Dort hat er auch einzelne Bände verfasst. And last, but not least: Er betreut die jährlich erscheinende Textausgabe im dtv «Energierecht». Insgesamt also: Neben der aufreibenden Arbeit in einer ständig wachsenden energierechtlich ausgerichteten Kanzlei eine beachtliche Leistung, die viel Tatkraft, Ideenreichtum und Disziplin verlangt.

Mit den neuen Herausforderungen des Energierechts hat sich inzwischen auch eine beachtliche Anzahl von Kommentaren in der Gelben Reihe entwickelt, die die zunehmende rechtliche und wirtschaftliche Bedeutung des Rechtsgebiets dokumentieren. Sie sind sämtlich darauf ausgerichtet, die immer komplexeren und juristisch zunehmend weniger fassbaren Normen des Energierechts kompakt, begrifflich und systematisch überzeugend und ausgerichtet auf die Fragestellungen der energierechtlichen Praxis erläutern. Dazu ist es gelungen, erfahrene Wissenschaftler zumeist aus energierechtlichen Instituten der Universitäten, aber auch bedeutende Praktiker insbesondere aus dem Mitarbeiterstab der Bundesnetzagentur und aus der Anwaltschaft zu gewinnen.

Hier die wichtigsten Beispiele der Gelben Kommentare: Gabriele Britz gibt gemeinsam mit den Professoren Johannes Hellermann und Georg Hermes den Kommentar zum Energiewirtschaftsgesetz heraus. Er ist erstmals 2008 erschienen, die 3. Auflage steht nun bevor. Schwerpunkte dieser Kommentierung sind das detailliert geregelte Regulierungsrecht, die Planfeststellung für Hochspannungs- und Gasversorgungsleitungen einschließlich der Wegenutzung sowie das energierechtliche Behördenverfahren. Das Regulierungsrecht wird inzwischen durch die komplexen Regelungen der Anreizregulierungsverordnung ergänzt und modifiziert. Dazu haben Bernd Holznagel und Raimund Schütz, die bereits als Herausgeber im IT- und Telekommunikationsrecht in Erscheinung getreten waren, einen umfassenden, sehr spezialisierten Kommentar herausgegeben. Der inzwischen nur noch von Rechtsanwalt Martin Altrock und Regierungsdirektor Volker Oschmann herausgegebene Kommentar Altrock/ Oschmann/Theobald, Erneuerbare Energien-Gesetz, der bald schon in 4. Auflage vorliegt, behandelt das wesentliche Kernstück der «Energiewende», die zunehmende Nutzung regenerativer anstelle fossiler Energieträger und des nun auslaufenden Atomstroms. Eine in der Praxis wichtige Variante zu den erneuerbaren Energien stellt der Einsatz von Wärme dar. 
Auch dazu gibt es ein Gesetz, das Erneuerbare-Energien-Wärmegesetz. Natürlich darf auch hier ein Kommentar nicht fehlen, den der Leiter der Forschungsstelle Umweltenergierecht an der Universität Würzburg Thorsten Müller und die beiden Mitarbeiter im Bundesministerium für Energie, Naturschutz und Reaktorsicherheit Volker Oschmann und Guido Wustlich herausgegeben haben. Und auch schon zum Netzausbaubeschleunigungsgesetz liegt ein praxisgerechter Kommentar vor. Herausgeber sind die auch wissenschaftlich ambitionierten Rechtsanwälte Siegried de Witt und Frank J. Scheuten.

\section{c) Compliance}

Bereits 1977 trat in den USA mit dem Foreign Corrupt Practices Act ein Bundesgesetz in Kraft, das Zahlungen und Wertgeschenke an ausländische staatliche Amtsträger als Gegenleistung für Geschäfte verbot. Seine ganze Brisanz für deutsche Unternehmen und die Buchführung (Schmiergelder waren in den seltensten Fällen überhaupt in den Büchern verzeichnet, und wenn, dann falsch) entfaltete das Gesetz allerdings nur, sofern diese in den USA börsennotiert waren. Da immer mehr deutsche Unternehmen den Gang an die Wall Street wagten, wuchs auch das Risiko, in den Fokus der amerikanischen Börsenaufsicht zu geraten. Die 1991 in Kraft getretenen und 2004, überarbeiteten US Sentencing Guidelines verlangten von Unternehmen, die in den USA Geschäfte machten, erstmals explizit ein «effektives» Compliance-Programm. Im Falle von Rechtsverstößen richtete sich die Höhe der Strafe ab jetzt u. a. danach, ob das Unternehmen ein solches «effektives» Compliance-Programm vorweisen konnte.

Auch in Deutschland änderte sich die Verfolgungspraxis: Während bis zum 1. Januar 1999 «nützliche Aufwendungen» steuerlich absetzbar waren und Unternehmen sogar auf Zahlungsbelege verzichten konnten, wurde dies ab sofort radikal anders. Plötzlich wurden Schmiergeldzahlungen als Wirtschafts- und Steuerstraftat verfolgt, viele Unternehmen mussten ihre Geschäftspraktiken von heute auf morgen umstellen. Mit dem am 15. Februar 1999 in Kraft getretenen Gesetz zur Bekämpfung der Internationalen Bestechung (IntBestG) wurde der Paradigmenwechsel in der Strafverfolgung sichtbar. Zwar stellte auch zuvor schon die Bestechung ausländischer Amtsträger eine Straftat dar. Die Verfolgungsintensität der zuständigen Behörden und das Bewusstsein in den Unternehmen waren aber noch anders.

Das Ordnungswidrigkeitenrecht hatte zwar schon immer in seinen $\mathbb{S} 9$, 30, 130 OWiG schuldhaft unterlassene Aufsichtsmaßnahmen seiner vertre- 
tungsberechtigten Organmitglieder sanktioniert, durch die spezialgesetzliche Normierung von Organisationspflichten in neueren Gesetzen (u. a. dem WpHG, dem BImSchG, dem KrW/AbfG oder dem GeldwäscheG) wurden die Pflichten aber nunmehr stärker konkretisiert. Es wurde damit immer wichtiger, die Einhaltung der Gesetze - insbesondere hinsichtlich potentiell korruptionsgeneigter Abteilungen und Auslandsniederlassungen - zu überwachen, die Mitarbeiter entsprechend zu schulen und dies auch zu dokumentieren, um im Fall einer Verfolgung durch die Strafverfolgungs- oder Steuerbehörden das Bemühen um eine ordnungsgemäße Unternehmensorganisation nachweisen zu können.

All dies firmierte fortan auch in Deutschland unter dem Begriff «Compliance». Die Stimmen, die zunächst das Sicherstellen der Einhaltung rechtlicher Regeln - so die einfache Umschreibung des Begriffs «Compliance» - als vorübergehenden «Lärm um nichts» und als «alten Wein in neuen Schläuchen» verstanden, verstummten recht bald angesichts der immer neuen aufgedeckten Skandale und der steigenden Personalkosten in den Compliance-Abteilungen der Unternehmen.

Auch das Haftungsrisiko von Unternehmensleitungen für eine fehlerhafte Unternehmensorganisation änderte sich. Unter dem Eindruck der ARAG/Garmenbeck-Entscheidung des Bundesgerichtshofs im Jahr 1997, die erstmals einen Aufsichtsrat zur Geltendmachung von erfolgversprechenden Ansprüchen gegen Vorstände zwingt, sobald er von haftungsauslösenden Sachverhalten Kenntnis erhält, wurde das Thema «Compliance» auch in den Führungsetagen großer deutscher Unternehmen virulent.

In der Folge wuchsen auch bei juristischen Beratern das Arbeitsaufkommen und damit das Bedürfnis nach maßgeschneiderter Literatur. Das Thema Compliance wurde aufgrund der häufig zwangsläufig auch strafrechtlichen Folgen mangelnder Unternehmensorganisation im Verlag bald an der Schnittstelle zwischen Gesellschafts- und Strafrecht verortet. Der Verlag konnte auch auf diesem publizistisch noch in den Kinderschuhen befindlichen Gebiet einen tatkräftigen Herausgeber und Autor gewinnen: Christoph Hauschka, nach beruflichen Stationen in Rechtsabteilungen von verschiedenen Unternehmen damals noch Justitiar eines bekannten Baukonzerns, hatte sich das Thema «Compliance» auf seine Fahne geschrieben und konnte den Verleger Hans Dieter Beck von der Verkäuflichkeit eines Handbuchs zum völlig neuen Thema Compliance überzeugen. Im Frühjahr 2006 erschien das von Hauschka herausgegebene Handbuch «Corporate Compliance» in erster Auflage. Dass zufällig nur wenige Monate zuvor in einer Aufsehen erregenden Aktion bei einem 
großen Unternehmen nach staatsanwaltlichen Ermittlungen bundesweit Durchsuchungen mit nachfolgend mehrjährigen Strafverfahren durchgeführt wurden und das Thema auch in den Folgejahren durch immer neue Bestechungsskandale und Kartellverfahren befeuert wurde, war für den Verkauf des Werkes alles andere als schädlich: 2010 erschien die zweite Auflage, die dritte Auflage ist in Vorbereitung.

Das Werk war Auftakt einer ganzen Serie von Compliance-Titeln, die das Lektorat in der Folge projektieren und realisieren konnte. Ab dem Jahr 2008 wurde der Bereich auch um eine eigene Zeitschrift, die «Corporate Compliance Zeitschrift - CCZ», ergänzt. Auch hierbei hatte der findige Christoph Hauschka, als Mitinitiator neben dem Münchener Rechtsanwalt Thomas Klindt, wieder einen günstigen Moment abgepasst, um den Verleger vom Bedürfnis und den Marktchancen einer solchen Publikation zu überzeugen. Zu Recht, wie sich herausstellen sollte: Andere Verlage zogen mit eigenen Zeitschriften und Büchern zum Thema Compliance nach.

\section{Neues auf dem Handbuchmarkt: Anwaltshandbücher und weitere Formularbuchreihen}

\section{a) Handbücher speziell für den Anwalt}

Die Entwicklung von Handbüchern speziell für den Rechtsanwalt begann 1987 mit dem von den Rechtsanwälten Hans-Ulrich Büchting und Benno Heussen herausgegebenen «Beck'schen Rechtsanwalts-Handbuch». Das Werk behandelt für zahlreiche Rechtsgebiete die Standardprobleme bei der jeweiligen Mandatsbearbeitung. Dieses Kompendium erfreut sich insbesondere auch bei Berufsanfängern nach wie vor großer Beliebtheit und ist für Beratungssituationen konzipiert, in denen der Rechtsanwalt außerhalb seiner Spezialdisziplinen tätig werden muss. Hans-Ulrich Büchting war neben seiner Tätigkeit als Rechtsanwalt langjähriger Lektor und Justitiar des Verlages C.H.Beck. Benno Heussen wurde 1973 in München als Rechtsanwalt zugelassen und gründete nach Mitarbeit in mehreren bekannten Kanzleien und Fusionen die Sozietät Heussen Rechtsanwälte als GmbH mit mehreren Standorten in Deutschland und Repräsentationsbüros in New York und Brüssel. 2003 wurde er in Hannover zum Honorarprofessor ernannt. Sein zweiter Schwerpunkt neben seinem primären Interesse an der Informationstechnologie (siehe dazu S. 493) liegt in den Bereichen Anwaltsmanagement und berufsständische Fragen. Deshalb 
war er acht Jahre im Vorstand des Deutschen Anwaltvereins (DAV) und gründete dort die Arbeitsgemeinschaften «Informationstechnologie» und «Kanzleimanagement».

Im Anschluss an das Beck'sche Rechtsanwaltshandbuch hat sich der Verlag, allerdings erst 12 Jahre später, entschlossen, mit der Reihe «Münchener Anwaltshandbuch» weitere, vertiefte Darstellungen zu einzelnen Rechtsgebieten anzubieten. Anlass für den Aufbau einer solchen Reihe war die zunehmende Spezialisierung der Anwaltschaft, die sich in der Einführung zahlreicher neuer Fachanwaltsbezeichnungen widerspiegelte. Bis 1999 beschrieb die Fachanwaltsordnung lediglich sieben Rechtsgebiete, für die man einen Fachanwaltstitel erwerben konnte. Mit der Jahrtausendwende begannen lebhafte Diskussionen der berufsständischen Organisationen zur Begründung weiterer Fachanwaltschaften. Bis heute wurde der Kanon auf 20 Rechtsgebiete ausgedehnt. Dazu zählen auch sehr spezielle Gebiete, wie etwa das Agrarrecht. Die Spezialisierung eines weiten Teils der Anwaltschaft erforderte «maßgeschneiderte» Konzepte. Kennzeichen der neuen Reihe war und ist die Integration von Checklisten, Formulierungsvorschlägen, Mustern und Beispielen in die systematische Darstellung.

Als erster Band in dieser Reihe ist im Jahre 2001 das «Münchener Anwaltshandbuch Straßenverkehrsrecht» von Hans Buschbell erschienen. Buschbell, dessen anwaltlicher Schwerpunkt im Straßenverkehrs- und Versicherungsrecht liegt, ist Senior in einer überörtlichen Sozietät in Düren und Köln. Er arbeitet eng mit den Rechtsschutzversicherungen zusammen und gilt als Erfinder der anwaltlichen Telefonberatung 24, Stunden rund um die Uhr.

Noch im selben Jahr folgten die Handbücher zum «Wohnraummietrecht» (später: «Mietrecht»), herausgegeben von den Rechtsanwälten Thomas Hannemann und Michael Wiegner, und zum «Erbrecht», herausgegeben von Stephan Scherer, Sozius der bekannten Mannheimer Kanzlei Schilling, Zutt \& Anschütz. Die Kernkompetenz von Thomas Hannemann liegt im privaten Immobilienrecht, im Bau- und Architektenrecht, im Bauträgerrecht, im Maklerrecht sowie im Miet- und Pachtrecht. Er ist Vorsitzender der Arbeitsgemeinschaft «Mietrecht und Immobilien» im Deutschern Anwaltsverein (DAV) sowie beliebter Referent insbesondere im Miet- und Immobilienrecht auf über 4,o Seminaren im Jahr. Das Interesse von Stephan Scherer liegt neben dem Erbrecht sowie der Unternehmensnachfolge insbesondere im Stiftungswesen, wo er sich in mehreren Verbänden stark engagiert. 
Auch zum gesamten «Gewerblichen Rechtsschutz» konnte im Jahr 2001 ein beachtliches Querschnittswerk, herausgegeben von Gordian Hasselblatt, ins Leben gerufen werden. Gordian Hasselblatt ist Partner im Kölner Büro der Kanzlei CMS Hasche Sigle und hat sich auf das Recht des geistigen Eigentums spezialisiert. Besonders im Markenrecht gehört er zu den führenden Rechtsanwälten in Deutschland.

Im folgenden Jahr 2002 gelang auch ein Durchbruch im Familienrecht: Unter der Herausgeberschaft des Kölner Anwalts Klaus Schnitzler wurde ein fachlich excellenter Autorenkreis zusammengestellt, der zwischenzeitlich an der 4. Auflage des «Münchener Anwaltshandbuchs Familienrecht» arbeitet. Der Verlag konnte im selben Jahr noch ein weiteres Münchener Anwaltshandbuch auf den Markt bringen, das den Arbeitsbereich der Fachanwälte für Verwaltungsrecht abdeckt. Für den entsprechenden Band zum Verwaltungsrecht konnte man den erfahrenen Kölner Anwalt Heribert Johlen und seinen Sozius Michael Oerder gewinnen.

Für den großen Bereich des Gesellschaftsrechts bestritt der Verlag nicht wie seine Konkurrenten den Weg eines Gesamtbandes für den Fachanwalt für Handels- und Gesellschaftsrecht, sondern initiierte drei Bände, aufgeteilt nach Gesellschaftsformen. So wurde zunächst Ende 2002 der Band zum «GmbH-Recht», herausgegeben von dem anwaltlichen «Multitalent» Volker Römermann, aufgelegt, dem folgten Ende 2004 der Band zum «Aktienrecht», herausgegeben von dem Stuttgarter Rechtsanwalt, Steuerberater und Wirtschaftsprüfer Matthias Schüppen und dem Münchner Notar Bernhard Schaub, und Anfang 2005 der Band zum «Personengesellschaftsrecht», herausgegeben von Hans Gummert.

Auch zum Arbeitsrecht, durfte - trotz dicht gedrängten Markts - natürlich in der Reihe kein Band fehlen. Wilhelm Moll, Sozius der bekannten Kanzlei Heuking Kühn Lüer Wojtek, hat sich zusammen mit zahlreichen Kollegen dieser Herausforderung gestellt und erstmals 2004, ein weiteres beachtliches Werk vorgelegt; zwischenzeitlich hat auch dieser Band die 3. Auflage erreicht.

Besonderer Erwähnung bedarf das 2006 erschienene «zweibändige» «Münchener Anwaltshandbuch für die Strafverteidigung», an dem 122 Autoren über fünf Jahre gearbeitet haben. Der erste von dem leider allzu früh verstorbenen Rechtsanwalt Gunter Widmaier herausgebrachte Band ist für die allgemeine Strafverteidigung konzipiert, der zweite von Klaus Volk herausgegebene Band dient speziell der Verteidigung in Wirtschafts- und Steuerstrafsachen. Gunter Widmaier gehörte zu den herausragenden Strafverteidigern der letzten zo Jahre mit einer eigenen, auf Revisionen und Ver- 
fassungsbeschwerden spezialisierten Kanzlei in Karlsruhe in unmittelbarer Nähe zum Bundesgerichtshof und Bundesverfassungsgericht. Widmaier leitete über zehn Jahre den Strafrechtsausschuss der Bundesrechtsanwaltskammer und wirkte an führender Stelle durch Gutachten und als Mitglied in der ständigen Deputation beim Deutschen Juristentag mit. Widmaier war an der Klärung zahlreicher wichtiger strafrechtlicher Rechtsfragen durch den Bundesgerichtshof und das Bundesverfassungsgericht beteiligt. Die Schwerpunkte des Münchner Professors Klaus Volk liegen vornehmlich im nationalen und internationalen Wirtschaftsstrafrecht sowie im Strafprozessrecht. Neben seiner Aufgabe als Hochschullehrer fand vor allem seine Tätigkeit als Strafverteidiger in spektakulären Verfahren, wie u. a. als Verteidiger von Josef Ackermann im Mannesmann-Prozess und von Boris Becker in dessen Steuerstrafverfahren, öffentliche Aufmerksamkeit.

Der Aufbau der Reihe der Münchener Anwaltshandbücher mit derzeit 22 Bänden in nur zehn Jahren mit zahlreichen Herausgebern und einer Vielzahl von Autoren stellt eine beachtliche inhaltliche, aber auch organisatorische Leistung des Verlages dar. Trotz der nicht unerheblichen Konkurrenz anderer Verlage, wie insbesondere Luchterhand, Dr. Otto Schmidt und Heymanns, hat die Reihe der Münchener Anwaltshandbücher bei der Anwaltschaft großen Beifall gefunden und sich weitgehend auch wirtschaftlich durchgesetzt. Besonders erfolgreich sind die Bände zum Erbrecht und zum Gewerblichen Rechtsschutz, die bereits in 4. Auflage erscheinen konnten. Als vorläufig letzten Band hat der Verlag im Jahre 2012 das von Andreas Fandrich und Ines Karper herausgegebene «Handbuch zum Bank- und Kapitalmarktrecht» veröffentlicht.

Mit ähnlicher Konzeption wie die Münchener Anwaltshandbücher, jedoch zu spezielleren Themen, die sich an den früheren «Interessen- und Tätigkeitsschwerpunkten» der Anwaltschaft orientierten, entwickelte der Verlag die Reihe «Beck'sches Mandatshandbuch». Sie wurde 2002 eröffnet und umfasst inzwischen 11 Bände.

Neben den durchweg in der typisch Beck'schen roten Farbe publizierten Handbüchern für die Anwaltschaft darf die NJW-Schriftenreihe - heute «NJW Praxis» - nicht unerwähnt bleiben, die schon seit den 1970er Jahren wichtige anwaltliche Themen aufgreift. Einige von ihnen haben im Laufe der Zeit den vorgesehenen Umfang weit gesprengt und sich zu echten Handbüchern entwickelt.

Beispielhaft genannt seien hier zwei besonders erfolgreiche Bände; zum einen der von Eugen Stahlhacke, Ulrich Preis und Reinhard Vossen bearbeitete Band «Kündigung und Kündigungsschutz im Arbeitsver- 
hältnis», dessen bereits 10. im Jahr 2010 erschienene Auflage über 9oo Seiten umfasst und zwischenzeitlich als Handbuch außerhalb der Reihe platziert werden konnte.

Zum anderen ist der von Elmar Kalthoener, ehemals Vorsitzender Richter am Oberlandesgericht Köln, begründete und über viele Jahre von Helmut Büttner, Richter ebenfalls an diesem Gericht, fortgeführte Band «Die Rechtsprechung zur Höhe des Unterhalts» zu erwähnen, der inzwischen zwölf Auflagen erlebt hat und nunmehr von Birgit Niepmann und Werner Schwamb bearbeitet wird.

Auch in der jüngeren Vergangenheit konnten zahlreiche Neuerscheinungen innerhalb der Reihe veröffentlicht werden, die besondere Tätigkeitsbereiche der Anwaltschaft aufgreifen, wie z. B. das Vergaberecht, die Mediation, Litigation im Gesellschaftsrecht oder den Anlegerschutz. Die Reihe umfasst heute weit über 70 lieferbare Titel und deckt 25 Rechtsgebiete ab.

\section{b) Formularbücher}

Die anhaltenden Erfolge der in den 1980er Jahren begründeten Formularbücher, so wie einige Nachahmungsversuche der Konkurrenz, veranlassten den Verlag, über den weiteren Ausbau dieser Buchgattung nachzudenken. In den bisher verlegten Werken konnten nicht annähernd alle Fallkonstellationen behandelt werden, so dass zwei neue Reihen entstanden: eine Reihe für Klage- und Schriftsatzmuster sowie eine Reihe für Vertragsmuster.

Für die forensische Tätigkeit wurde die neue Reihe «Münchener Prozessformularbuch» geschaffen, die die Tradition des «Beck'schen Prozessformularbuchs» fortsetzen und in einzelnen Teilbänden verbreitern sollte. Die Reihe war in erster Auflage auf sechs Bände angelegt. Als erstes Werk erschien 1998 der von Rechtsanwalt Heribert Johlen herausgegebene Band zum «Verwaltungsrecht», der inzwischen drei Auflagen erlebt hat. Die weiteren fünf Bände erschienen in den folgenden drei Jahren in 1. Auflage: zum «Mietrecht», herausgegeben von Paul Jendrek, später von Ulf Börstinghaus; zum «Privaten Bau- und Architektenrecht», begründet von Wolfgang Koeble und Rolf Kniffka. Das «Familienrecht» gab Peter Gottwald heraus und das Prozessformularbuch zum «Gewerblichen Rechtsschutz» Peter Mes. Schließlich erschien das «Arbeitsrecht», herausgegeben von Ulrich Zirnbauer.

Als die 2. Auflage dieser Bände anstand, wurde die Reihe um einen weiteren Band, «Erbrecht», ergänzt; der Münchner Anwalt Bernhard Klinger gibt ihn heraus. Er ist auch durch zahlreiche andere Veröffentlichungen bekannt. 
Besonders erfolgreich sind folgende, bereits in 4. Auflage befindlichen Bände: Familienrecht, dessen Herausgeber der dem Verlag aus anderen Werken eng verbundene und an der Universität Regensburg lehrende Prozessualist Peter Gottwald ist. Er hat die Struktur des familienrechtlichen Prozessformularbuchs ausgearbeitet. Der Nürnberger Fachanwalt für Arbeitsrecht Ulrich Zirnbauer als Betreuer des arbeitsrechtlichen Prozessformularbuches ist nicht nur als Herausgeber und Autor zahlreicher Fachbücher auf dem Gebiet des Arbeitsrechts, sondern auch in berufsständischen Organisationen, im Vorstand des Deutschen Anwaltsvereins und der Rechtsanwaltskammer Nürnberg stark engagiert. Beim Privaten Bauund Architektenrecht hat der Reutlinger Rechtsanwalt Wolfgang Koeble die Federführung. Er ist Autor und Herausgeber zahlreicher anderer Werke, wie z. B. des «Rechtshandbuchs Immobilien».

Ab 2001 hat der Verlag mit der weiteren Reihe der «Beck'schen Formularbücher» die Anwaltsliteratur im Bereich der kautelarjuristischen Formularwerke noch weiter ausgebaut. In bisher 13 auf einzelne Rechtsgebiete bezogenen Bänden werden dem Anwalt zahlreiche spezielle Vertrags- und Erklärungsmuster mit einer vertieften Erläuterung zur Verfügung gestellt. Die Reihe wurde mit dem Band zum «Immobilienrecht» von Stefan Weise eröffnet, der in Kürze in stark überarbeiteter Form eine Neuauflage erfahren wird.

Besonders erfolgreich sind in dieser Reihe die bereits in 3. Auflage erschienenen Bände zum «Familienrecht», herausgegeben von dem Münchner Familienrechtsanwalt Ludwig Bergschneider, zum «IT-Recht», herausgegeben von Wolfgang Weitnauer, und zum «Mietrecht», herausgegeben von dem zwischenzeitlich leider viel zu früh verstorbenen Rechtsanwalt Günter Nies, u. a. Mitherausgeber der Beck'schen mietrechtlichen Zeitschrift NZM, und dem Kölner Richter Richard Gies.

Konzeptionelles Neuland betrat der zwischenzeitlich in 2. Auflage vorliegende Band zum Zivil-, Wirtschafts- und Unternehmensrecht, der alle enthaltenen Vertragsformulare in deutscher und englischer Sprache synoptisch gegenüberstellt, und der mit einer einheitlichen Terminologie über sogenannte Wortlisten Standards für die Vertragsgestaltung setzt. Herausgegeben wird dieser Band vom Münchner Notar Robert Walz.

Neben den geschilderten Hand- und Formularbüchern gibt es zu ganz speziellen Rechtsproblemen noch zahlreiche kürzere Darstellungen, deren auch nur beispielhafte Aufzählung den Rahmen sprengen würde. Insgesamt wurde so mit einer speziell auf den Rechtsanwalt zugeschnittenen 
Literatur eine neue Programmsparte geschaffen, die durch zahlreiche Titel getragen wird.

\section{Rote Kommentarreihe}

Das Flaggschiff des Verlages, der Münchener Kommentar zum BGB, folgte im Laufe der Jahrzehnte weiter seiner erfolgreichen Bahn. Daneben suchten zahlreiche junge Wissenschaftler und Habilitanden nach neuen Herausforderungen. Aber in Beck'schen Werken zum BGB gab es nur selten vakante Kommentierungsabschnitte. Mit schöpferischer Phantasie entdeckte man im Verlag eine Lücke. Zwischen dem einbändigen Palandt und dem jetzt schon elfbändigen Münchener Kommentar zum BGB stand nur der zweibändige Erman, der vom Aschendorff-Verlag in den Otto Schmidt-Verlag wechselte. Die Lücke existierte nicht nur vom Umfang her. Vielmehr fehlte auch konzeptionell ein Kommentar, der auf der einen Seite in einer gewissen Ausführlichkeit die dogmatischen Grundlagen herausarbeitete und auf der anderen Seite in aller Kürze die für die Praxis wesentliche Rechtsprechung zusammenfasste.

In den ersten Überlegungen, die durch eine Synopse der bestehenden BGB-Kommentare - Jauernig, Palandt, Erman, Münchener Kommentar, Soergel und Staudinger - untermauert wurde, galt es, eine Entscheidung zum gewünschten Inhalt zu fällen. Ein wirtschaftlich ausgerichteter Kommentar ohne Familien- und Erbrecht in zwei Bänden wurde diskutiert, aber letztendlich verworfen. Viele Fragen, etwa zur Fortführung der Gesellschaft, zur Geschäftsfähigkeit oder zur Vertretung, sind ohne Familienund Erbrecht nicht zu lösen. Auch Probleme der Wirkungen der Ehe im Allgemeinen, man denke nur an die Bestimmung zu den Geschäften zur Deckung des Lebensbedarfs, sind ohne Familienrecht nicht zu entscheiden. Dennoch sollte der neue Kommentar mit zwei Bänden einen Schwerpunkt auf die ersten drei Bücher des BGB setzen, Familien- und Erbrecht sollten ebenso knapp gehalten werden wie das Internationale Privatrecht. Als Zielgruppe hatte der Verlag den Praktiker im Auge, der täglich mit der raschen Lösung von Rechtsfragen befasst ist und gelegentlich einmal bisher noch nicht höchstrichterlich entschiedene Probleme dogmatisch vorangehend einer praktikablen Lösung zuführt.

Mit den beiden Herausgebern Georg Bamberger, damals Präsident des Oberlandesgerichts Koblenz, und Herbert Roth sowie damals 4, heute 72 Autoren war ein Team gewonnen, das sowohl die Praxis als auch die 
Wissenschaft repräsentierte. Die Begeisterung bei den angefragten Autoren war groß, manchmal von einer gesunden Skepsis begleitet, ob es gelingen würde, ein komplettes Autorenteam zusammen zu stellen, das in angemessener Zeit die Manuskripte fertig stellen würde. Der Arbeitstitel für das neue Werk war Programm: BGB 2ooo. Damit war definiert, dass sich Herausgeber, Autoren und Verlag das Ziel gesetzt hatten, nach einer Entstehungszeit von drei Jahren zur Jahrtausendwende einen neuen zukunftsweisenden Kommentar vorzulegen.

Die optimistischen Planungen hatten gewisse Verzögerungen beim Fertigstellen der Manuskripte und die Notwendigkeit, Autoren bei ihrer mühsamen Arbeit zu entlasten oder durch andere zu ersetzen, einkalkuliert. Die Verzögerungen gingen aber über das Erwartete hinaus. Lag es an der erwarteten Schuldrechtsmodernisierung?

Über diese Schuldrechtsmodernisierung war schon viele Jahrzehnte diskutiert worden, ohne dass es zu einem Gesetzesbeschluss gekommen wäre. In der 14. Legislaturperiode (1998-2004) war die Zeit dann endlich reif für den großen Wurf. Überlegungen, den Kommentar trotz Schuldrechtsmodernisierung nach altem Recht zu publizieren, scheiterten daran, dass noch nicht alle Manuskripte geschrieben waren. Auch eine Veröffentlichung als Online-Produkt kam deshalb nicht in Betracht. Aus BGB 2000 wurde der Bamberger/Roth, Kommentar zum Bürgerlichen Gesetzbuch, erschienen von Oktober 2002 bis April 2003 in drei Bänden mit einem Gesamtumfang von rund 8000 Seiten. Der Bamberger/Roth war damit der erste mehrbändige Kommentar, der komplett zur Rechtslage nach der Schuldrechtsmodernisierung vorlag. Rückblickend war es die richtige Entscheidung, einen neuen Kommentar nicht mehr zum alten Recht zu publizieren. Damit gewann der Bamberger/Roth eine hervorragende Ausgangsposition für seine weitere Entwicklung. Die auf die Entstehungszeit zurückreichende Idee, von vornherein eine elektronische Verwertung vorzusehen, wurde weiter ausgebaut. Nicht mehr die Verfügbarkeit des unveränderten Printwerkes als Online-Produkt, sondern die Weiterentwicklung zu einem Beck'schen Onlinekommentar mit vierteljährlichen Aktualisierungen wurde realisiert. Siehe dazu auch unten S. $5^{21}$ ff. Nach der 2. Auflage 2007/2008 mit einem Gesamtumfang von 9200 eng bedruckten Seiten ist 2012 die 3. Auflage des Printwerkes mit 954,2 Seiten erschienen.

Es folgten weitere Kommentare in der Ausstattung des «Bamberger/ Roth.» Auch der zweibändige Kommentar zum Aktiengesetz, herausgegeben von Eberhard Stilz, Präsident des Oberlandesgerichts Stuttgart, und Gerald Spindler, sollte eine Position zwischen dem einbändigen Hüffer in 
der Reihe der Kurz-Kommentare und dem großen Münchener Kommentar einnehmen. Das ursprüngliche Team war mit zehn Autoren klein gehalten. Innerhalb von vier Jahren war die Manuskriptabgabe anvisiert. Keine großen Reformen, aber zahlreiche Änderungen - Einfügung der $\$ \$ 327$ a bis $3^{27} \mathrm{f}$ zum Squeeze out, das Spruchverfahrensneuordnungsgesetz, der neue $\$$ 161 AktG und der Corporate Governance Kodex, Rechnungslegung und IAS, die Auswirkungen der Übernahmerichtlinie und die SE-VO - und das Ausscheiden einiger Mitwirkender erforderten neue Planungen. Diesmal wurde die Zeit bis zur Manuskriptablieferung knapper berechnet, um nicht nochmals Gefahr zu laufen, dass die eingegangene Verpflichtung zu weit in künftige Aufgaben verschoben würde. Auch war der Arbeitsabschnitt, den ein Autor zu bearbeiten hatte, in der Regel weniger umfangreich als ursprünglich. So wurden aus den zehn nun 4,1 Autoren. Im September 2007 hatte die 1. Auflage schließlich den Weg zum Buchhändler geschafft - rechtzeitig vor der Konkurrenz. Die 2. Auflage folgte 2010, nun schon auf einem dichter besetzten Markt an aktienrechtlicher Literatur.

Der einbändige Kommentar zum Handelsgesetzbuch, herausgegeben von Hartmut Oetker, ist in 1. Auflage 2009, in 2. Auflage 2011 und im Juni 2013 kurz nach der Seehandelsrechtsreform in 3. Auflage erschienen. Ein kleines Autorenteam und ein effektiver Herausgeber aktualisiert das Werk zuverlässig und zeitnah.

Als weiterer gesellschaftsrechtlicher Kommentar ist das zweibändige Werk zum GmbHG, herausgegeben von Lutz Michalski, 1. Auflage 2002 und 2. Auflage 2010, zu nennen. Hinzuweisen ist insbesondere auf die ausführliche systematische Einleitung mit Ausführungen zum Internationalen Gesellschaftsrecht, zur Besteuerung der GmbH, zum Konzernrecht in der GmbH und zur Finanzierung. Professor Lutz Michalski, Jahrgang $195^{\circ}$ promovierte 1978 in Münster bei Professor Harry Westermann zum Thema «Gesellschaftsrechtliche Gestaltungsmöglichkeiten zur Perpetuierung von Unternehmen». Seine Habilitation folgte 1987 an der Universität Bielefeld wiederum zu einem gesellschaftsrechtlichen Thema: «Das Gesellschaftsund Kartellrecht der berufsrechtlich gebundenen freien Berufe». Sein literarisches Werk umfasst neben der Herausgeberschaft des GmbH-Kommentars die Kommentierung im Erman zu den $\$ \$ 9$ ff., $\$ \$ 929$ ff., $\$ \$ 10 z 0$ ff. und $\$ S 1611$ ff. BGB sowie weitere diverse Veröffentlichungen aus dem Bereich Arbeitsrecht, Erbrecht und zum Partnerschaftsgesellschaftsgesetz. Ferner hat er am Aufbau der Neuen Zeitschrift für Gesellschaftsrecht (NZG) maßgeblich mitgewirkt. Von 1987 bis zu seinem Tod im Mai 2013 war er Professor an der Universität Bayreuth. 
Nach der UWG-Reform 2004, waren die Programmplätze in der Reihe der Kurz-Kommentare und der Gelben Reihe bereits durch eingeführte Werke belegt. Für den Münchener Kommentar zum Lauterkeitsrecht sowie für den großen einbändigen Kommentar der Herausgeber Harte-Bavendamm/ Henning-Bodewig waren Autorenteams akquiriert. Der neue Kommentar zum Gesetz gegen den unlauteren Wettbewerb (UWG), herausgegeben von Karl-Heinz Fezer, wurde in der Reihe der roten Kommentare vorgesehen und hat dort in 1. Auflage 2005 und 2. Auflage 2010 seinen Platz erobert.

Die Reihe der roten Kommentare hat sich ständig weiter entwickelt. In dieser Ausstattung sind ferner erschienen: Sachs, Grundgesetz (siehe S. 391), Calliess/Ruffert, EUV/AEUV (siehe S. 404), Wandtke/Bullinger, Urhebergesetz, Weyand, Vergaberecht, und Kniffka, Bauvertragsrecht.

\section{Rechtsliteratur für Nichtjuristen}

Nicht vergessen werden darf, dass neben den Fachinformationen für Juristen auch ein umfangreiches Programm wie die Ratgeberliteratur für Verbraucher veröffentlicht wird. Traditionell waren dies die Beck-Rechtsberater im dtv, angeführt durch die populären Bände von Schaub, Arbeitsrecht von A - Z und Meine Rechte als Arbeitnehmer. Letztlich wurden mit diesem Programm fast alle wichtigen Gebiete in den Bereichen Mietrecht, Familienrecht und Verkehrsrecht ebenso wie in entlegeneren Bereichen abgedeckt. Die Änderungen der Lesegewohnheiten und die Tatsache, dass sich Verbraucher inzwischen vermehrt online informieren, führten zu einem Rückgang in diesem Bereich, so dass der Verlag neue Antworten auf die Bedürfnisse seiner Leser finden musste.

Dies waren zum einen die in DIN-A-4,-Format mit Klammerheftung erscheinenden Broschüren zu zentralen Rechtsfragen der privaten Lebensgestaltung. Allen voran ist hier die Broschüre «Vorsorge für Unfall, Krankheit und Alter» zu nennen, die zusammen mit dem Bayerischen Staatsministerium der Justiz und für Verbraucherschutz herausgegeben und entwickelt wurde. Die Broschüre erschien zunächst im Selbstverlag des Justizministeriums und erstmals 2004 bei C.H.Beck. Sie erklärt dem Laien in übersichtlicher und verständlicher Form alles über Betreuung, hält für diese Akte rechtlich geprüfte Formulare, die individuell ausgefüllt werden können und allseits akzeptiert werden.

Ein weiterer erfolgreicher Titel aus dieser Reihe ist seit 2009 «Vorsorge 
für den Erbfall». Im Hinblick auf die strengen Formvorschriften des bürgerlichen Rechts können hier zwar keine herausnehmbaren Formulare angeboten werden, die Broschüre enthält aber zahlreiche Formulierungsvorschläge für Testamente in den verschiedenen Familien- und Lebenssituationen, weist den Leser aber zugleich darauf hin, wo es für den Laien zu kompliziert wird und der fachlich fundierte Rat eines Steuerberaters, Rechtsanwalts oder Notars gesucht werden muss. Ergänzt werden diese zentralen Titel durch ein reichhaltiges Programm zu benachbarten Themen, wie beispielsweise Spezialfragen der Patientenrechte, Fragen der Führung der Betreuung, zum Mietrecht, zum Vereinsrecht usw. Diese Broschüren wurden stets zum Hartgeldpreis angeboten und kosten derzeit $4,90 €$.

Daneben entwickelte sich in einem ganz anderen Format, nämlich 10,4 X 16,1 cm, die Broschürenreihe Beck kompakt. Zu den erfolgreichsten Titeln dieser Reihe gehören: Pilz, Bilanzen lesen und verstehen, Kruell, Smaltalk, und Stackelberg, Selbstbewusstsein. Hier findet der Leser zu juristisch angehauchten Themen, insbesondere aber auch zu betriebsund volkswirtschaftlichen Fragestellungen sowie zu den sogenannten «Softthemen» praktische Hilfe. Fast noch mehr als die DIN-A-4-Broschüren kommen diese sehr kurz gefassten Informationen den modernen Bedürfnissen nach rascher, zielgenauer und zugleich umfassender Information entgegen.

\section{BeckAkademie Seminare}

Mit außerhalb seines Kerngeschäfts liegenden Formen der juristischen Wissensvermittlung hat sich der Verlag lange zurückgehalten. Immerhin wurden bereits seit 1978 «Beck-Seminare» durchgeführt, zuerst zusammen mit einem englischen Unternehmen, das die Idee an den Verlag herangetragen hatte, aber bald schon allein. Seitdem gab es regelmäßig Seminarveranstaltungen, wenn auch nur in überschaubarer Zahl. Ein gewisser Schwerpunkt bildete sich bei arbeitsrechtlichen Themen; dort hatte man die höchsten Teilnehmerzahlen erreicht. Zugpferde waren damals Eugen Stahlhacke, dessen Buch «Kündigung und Kündigungsschutz im Arbeitsverhältnis» zu dieser Zeit noch in der NJW-Schriftenreihe erschien, und das Team um Karl Fitting mit betriebsverfassungsrechtlichen Fragestellungen.

Ein zusammenhängendes Seminarprogramm oder eine spezielle Semi- 
narabteilung bestanden jedoch noch nicht. Geplant und organisiert wurde das Seminargeschäft von einem damit beauftragten Lektor, der das neben den ihm anvertrauten Büchern leisten musste. Initialzündungen für Seminare waren oft Buchneuerscheinungen oder Neuauflagen. Es ging nicht primär um ein neues, wirtschaftlich vielversprechendes Betätigungsfeld, sondern um eine Ergänzung der Buchproduktion, die von manchen Autoren als zusätzlicher Honorarerwerb geschätzt wurde und dem Verlag nützlich schien, weil so die Autorenbindung intensiviert, ein Kontakt vom Lektorat auch zu Endkunden hergestellt und schließlich in vereinzelten Fällen auch einmal neue Themen oder potentielle Buchverfasser getestet werden konnten, bevor Verlagsverträge abgeschlossen wurden.

Damals wie heute waren die meisten Seminare Tagesveranstaltungen, die in zentral gelegenen Hotels stattfanden. Es gab noch keinen Seminarkatalog. Geworben wurde allein mit Direktwerbebriefen und Anzeigen in Beck'schen Zeitschriften. Flyer zu den Werbebriefen erschienen puritanisch in schwarz/weiß. 1986 war das Seminarangebot trotzdem schon auf 17 Veranstaltungen angewachsen. Erstmals kam für arbeitsrechtliche Seminare die Bezeichnung «NZA-Seminare» auf, nachdem 1984, diese neue Zeitschrift erfolgreich gestartet war. So wurde das im Vergleich zum Buch «schnellere Medium» mit Seminaren kombiniert. Andere Zeitschriften folgten diesem Vorbild. So gab es später etwa auch «NJW-Seminare».

Die Deutsche Einheit im Oktober 1990 eröffnete den Beck-Seminaren ein neues Geschäftsfeld, bestand in den neuen Bundesländern doch ein enormes Bedürfnis nach professioneller Vermittlung von Kenntnissen im bundesdeutschen Recht. So wurden die «Beck-DDR-Seminare - Das Recht der Bundesrepublik Deutschland» aus der Traufe gehoben. Themen waren u. a. Steuer- und Bilanzrecht, Betriebsverfassungs- und Tarifrecht sowie Werkvertrags- und AGB-Recht. Zwar musste bei diesen Seminaren auf ein Mittagessen und die sonst so beliebten Kaffeepausen verzichtet werden. Dafür war die Seminargebühr mit 85,- DM zzgl. 14 \% MwSt. unschlagbar günstig. Die Zahlung wurde laut Prospekt übrigens noch durch Scheck erbeten, den heute schon kaum jemand mehr kennt.

Doch nicht nur auf dem Gebiet der ehemaligen DDR bestand Fortbildungsbedarf. Auf Grund der zunehmenden Gesetzesflut, vor allem bedingt durch europarechtliche Vorgaben, wuchs im gesamten Bundesgebiet die Nachfrage nach juristischen Fachseminaren. Zudem wurde in der Fachanwaltsordnung (FAO) eine sanktionierte Fortbildungspflicht verankert. Dies belebte den gesamten Seminarmarkt. Insbesondere das Deutsche Anwaltsinstitut (DAI) und die Deutsche Anwaltsakademie (DAA) bauten 
ihr Seminarangebot aus. Zudem kamen etliche neue Seminaranbieter auf den Markt. Auch bei den «Beck-Seminaren»stieg die Zahl der Veranstaltungen deutlich an: 1992 wurden bereits 70 Seminare angeboten. Zwar gab es immer noch keine verlagseigene Seminarabteilung. Dennoch wurden Konzeption und Organisation der Seminare nun zentral in die Hände eines eigens dafür bestimmten Lektors samt Sachbearbeiterin gelegt. Dies war eine wichtige Basis für das weitere Wachstum der «Beck-Seminare». Mit den «Beck'schen Steuerseminaren», die damals noch von der steuerrechtlichen Redaktion konzipiert wurden, erweiterte sich das Angebot nochmals.

Dann aber wurden so viele Veranstaltungen angeboten, dass 1995 der erste Seminarkatalog erschien. Verglichen mit dem heutigen Gesamtprogramm war dieser Katalog jedoch recht überschaubar. So zählte dieser gerade einmal 24 Seiten (zum Vergleich: Aktuelles Gesamtprogramm: 280 Seiten).

Neben Seminaren entwickelten sich als weiteres wichtiges Format größere Fachtagungen zu aktuellen rechtlichen Themen. Diese entstanden in Kooperation mit den Zeitschriften-Redaktionen. 1997 wurde erstmals eine «ZEV-Fachtagung» veranstaltet, die in diesem Jahr zum 17. Mal stattfindet. Die NZI-Jahrestagung wurde zwei Jahre später begründet und findet im Herbst zum 15. Mal statt. Auch die NZA- bzw. die IStR-Jahrestagung haben inzwischen eine längere Tradition. Vor allem bei den großen Tagungen zeigt sich, dass für die Teilnehmer mittlerweile nicht mehr alleine die reine Wissensvermittlung im Vordergrund steht. Die Jahrestagungen werden vor allem auch zur Kontaktpflege und zum wichtigen Netzwerken genutzt. Auch direkter Austausch und Diskussion mit Kollegen und angesehenen Experten sind wichtige Faktoren. Damit kommen insbesondere die Tagungen dem gewachsenen Kommunikationsbedürfnis in der Anwaltschaft nach.

2001 entstand eine selbständige Seminarabteilung mit eigenem Abteilungsleiter. Im ersten Halbjahr 2002 erschien zum ersten Mal ein Seminarkatalog in Farbe. Die Themenbereiche waren inzwischen auf neun angewachsen und reichten von A wie Arbeitsrecht bis Z wie Zivilrecht. Im Jahr 2005 wurden bereits 213 Seminare und Tagungen durchgeführt. Eine neue Internetseite mit einem Seminar-Shop ging 2008 online. Seitdem können Seminare auch direkt im Internet gebucht werden. Da der Verlag in der Zwischenzeit auch andere Seminaranbieter erworben hatte, wurde aus Gründen eines einheitlichen Auftritts die «BeckAkademie» als Dachmarke geschaffen. In diesem Zusammenhang änderte sich 2009 der Name der Seminarabteilung von «Beck-Seminare» in «BeckAkademie Seminare». 
Zwar ist die Nachfrage nach juristischen Fortbildungen im Laufe der Zeit weiter angewachsen, jedoch ist der Seminarmarkt inzwischen hart umkämpft. Es stiegen nicht nur das Fortbildungsbedürfnis, sondern auch die Zahl der Seminaranbieter. Ein wichtiger Mehrwert der Beck'schen Seminare ist neben erstklassigen Referenten und umfangreichen Unterlagen vor allem auch der sog. «Beck-Bonus»: So erhalten die Teilnehmer zusätzlich zum Seminar ein Exemplar hilfreicher Fachliteratur aus dem Verlagsangebot bzw. eine dreimonatige Freischaltung für ein passendes beck-online Fachmodul.

Die Teilnehmerstruktur hat sich mit der Zeit verändert. Zu Beginn wurden die Seminare ausschließlich von Rechtsanwälten bzw. Fachanwälten besucht. Inzwischen ist auch eine sehr große Anzahl von Unternehmensjuristen zu verzeichnen. Selbst Nichtjuristen, für die es sogar eigens konzipierte Veranstaltungen gibt, besuchen mittlerweile die Beck'schen Seminare.

2011 fanden erstmals die Sommerlehrgänge statt. Bei dieser neuen Veranstaltungsform handelt es sich um mehrtägige Fortbildungen, die in entspannter Atmosphäre an attraktiven Orten stattfinden. Die Teilnehmer haben so die Möglichkeit zur Urlaubszeit Fortbildung und Freizeitaktivitäten zu kombinieren.

Aktuell besteht das Seminarprogramm aus rund 400 Veranstaltungen, die in 20 verschiedenen Rechtsbereichen angeboten werden. Die wichtigsten Gebiete sind neben dem Arbeits-, Gesellschafts- und Steuerrecht vor allem das Vertragsrecht. Aber auch neue Rechtsbereiche sind entstanden. So komplettieren inzwischen Veranstaltungen zum Energierecht und zu Compliance das Angebot. Neben den zahlreichen Seminaren finden aktuell jährlich 15 Tagungen und über 20 Sommerlehrgänge von «AGB und Vertragsklauseln» bis hin zu «Umwandlungssteuerrecht» statt.

\section{Die neue Form des Kommentars: der BeckOK (Beck'scher Online-Kommentar)}

Ab 2001 online gestellte Printwerke des Verlages gaben bald Anlass zu Überlegungen, ob daneben eine eigene Darstellungsform entwickelt werden müsse, die über die Grenzen des gedruckten Buches hinausgeht und alle Möglichkeiten des elektronischen Mediums konsequent ausnützt. Ziele waren dabei, aktueller als Printmedien zu sein, eine perfekte Verlinkung und die Darstellung in mehreren Ebenen. Im Hinblick auf diese Gesichtspunkte wurde die Konzeption für die Beck'schen Onlinekommentare entwickelt. 
Anders als die Printkommentare, die in Abständen von 1, 2, 3, manchmal bis zu 5 oder 6 Jahren erscheinen, werden die Onlinekommentare alle drei Monate aktualisiert. Die besonderen Möglichkeiten des Onlinemediums und des Bildschirms schaffen neben dem Standardtext noch eine mit der gesamten Kommentierung verknüpfte Überblicksebene, die jeweils die Grobstruktur der Norm wiedergibt. Zu ausgewählten Punkten gibt es ferner eine Detailebene - über den Button «Details» erreichbar - in der Kasuistik, komplexe rechtliche Streitfragen, aber auch Rechtstatsächliches oder Historisches referiert werden können, ohne den Standardtext als Hauptkommentierung zu sehr zu belasten und den Lesefluss abzulenken. Diese Darstellungsform, die heute auf Homepages üblich ist, wird so für die juristischen Fachmedien nutzbar gemacht.

Die Onlinekommentare stellen besondere Anforderungen an seine Autoren. Erstens erfordern sie die Bereitschaft, sich ständig mit dem Thema zu befassen, um so auch laufend Aktualisierungen liefern zu können. Zum anderen müssen die Texte der Autoren den Erfordernissen der Bildschirmansichten in besonderer Weise gerecht werden, keine zu langen Absätze enthalten und umgekehrt die Technik einer Darstellung in mehreren Ebenen nutzen.

Hans Dieter Beck selbst griff im Herbst 2003 die schon älteren Überlegungen des Lektorats für elektronische Publikationen zu den Beck'schen Onlinekommentaren auf. Beginnen wollte man im Arbeits- und Sozialrecht. Mit den jungen Hochschullehrern Professor Christian Rolfs, jetzt Köln, und dem Geschäftsführer bei der Deutschen Rentenversicherung, Professor Ralf Kreikebohm, hat der Verlag zwei Herausgeber gefunden, die bereit waren, die Idee aufzunehmen, weiterzuentwickeln und im Rahmen des Projektes eines Beck'schen Onlinekommentars - zu diesem Zeitpunkt noch kombiniert - zum Arbeits- und Sozialrecht umzusetzen. Professor Richard Giesen, jetzt München, und der damalige Vorsitzende Richter am Bundessozialgericht Professor Peter Udsching kamen als weitere Herausgeber hinzu. Im Sommer 2004, wurde der Herausgeberverlagsvertrag geschlossen und in der Folgezeit ein schlagkräftiges, überwiegend junges Autorenteam aus Wissenschaftlern und Praktikern zusammengestellt. Am 15. März 2006 ging der Beck'sche Onlinekommentar zum Sozialrecht ans Netz, mit einiger Verzögerung folgte am 13. Juli 2006 der arbeitsrechtliche Teil.

Doch blieb es nicht bei diesem Projekt. Auf Wunsch des Verlegers wurde im Mai 2004, eine Gesamtkonzeption für alle Rechtsgebiete entwickelt. Noch 2004, wurden weitere Herausgeber- und Autorenverträge geschlos- 


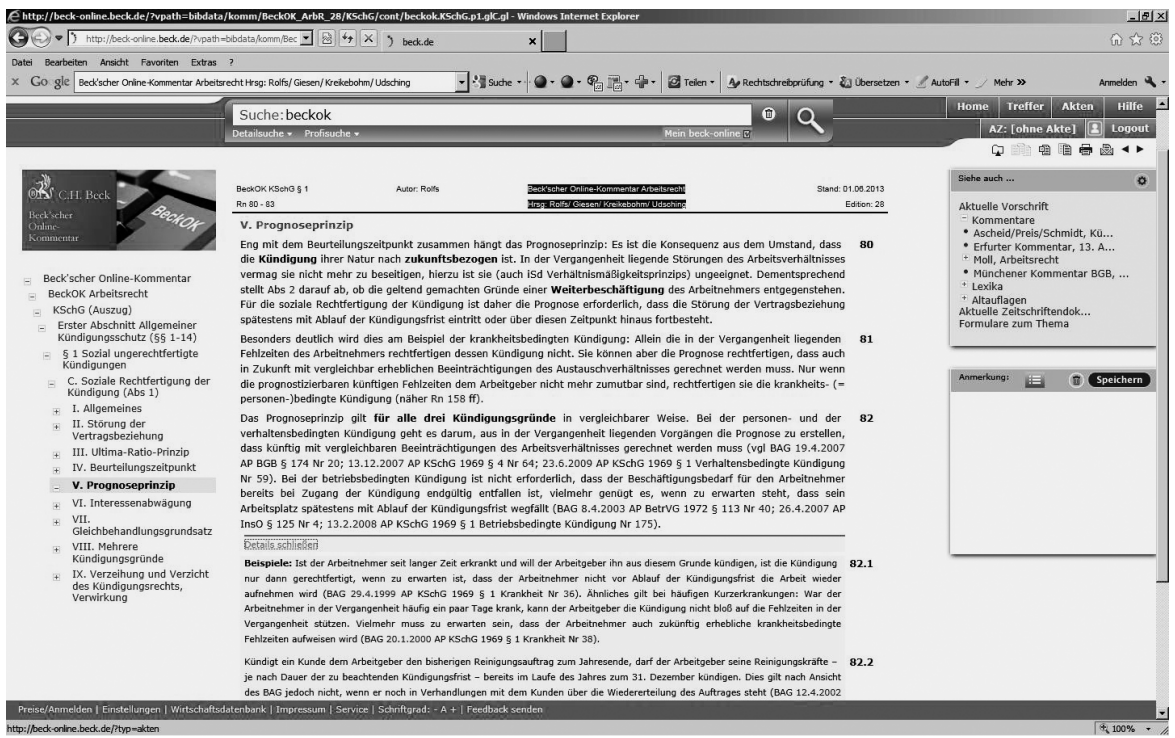

Beck'scher Online-Kommentar Arbeitsrecht, Hrsg: Rolfs/ Giesen/ Kreikebohm/ Udsching, Stand: 1. Juni 2013, Ed.: 28

sen. So folgten mit Livestellung am 18. Mai 2006 ein Beck'scher Onlinekommentar zur VOB B, herausgegeben von Professor Mathias Preussner, Konstanz, am 21. Juni 2006 ein Beck'scher Onlinekommentar zum Umweltrecht, herausgegeben von Rechtsanwalt Ludger Giesberts, Köln, und Professor Michael Reinhardt, Trier, am 20. Juli 2006 ein Beck'scher Onlinekommentar zur Grundbuchordnung, herausgegeben von Notar Professor Stefan Hügel, Weimar, und am 18. Oktober 2006 ein Beck'scher Onlinekommentar zum Strafgesetzbuch unter der Herausgeberschaft von Professor Bernd von Heintschel-Heinegg, Straubing.

Nicht nur zu zentralen Gebieten, sondern auch zu abgelegeneren Gebieten werden Beck'sche Onlinekommentare veranstaltet. So war im März 2006 zeitgleich mit dem Beck'schen Onlinekommentar zum Sozialrecht bereits der Beck'sche Onlinekommentar zum Tarifvertrag für den Öffentlichen Dienst (TVöD) in beck-online verfügbar. Dieses Werk hatte die Besonderheit, dass es - gleichsam wie ein Kommentar der Gesetzgebungsreferenten - von den maßgeblichen Repräsentanten der Tarifvertragsparteien herausgegeben wurde. In der ersten Edition waren dies der zuständige Vertreter der kommunalen Arbeitgeber Thomas Böhle von der Landeshauptstadt München, Frank Stöhr vom Deutschen Beamtenbund Tarifunion und Kurt Martin von der Gewerkschaft ver.di. Über den Sozial- 
parteien wacht der Vorsitzende Richter am Bundessozialgericht, jetzt a. D., Professor Klaus Bepler über die Ausgewogenheit des Werkes.

Eine Reihe von Onlinekommentaren erfuhren auch Ausdrucke als Printkommentare, so z. B. der zitierte BeckOK Umweltrecht, ohne dass dadurch die Onlineausgabe als führendes Medium in Frage gestellt worden wäre. Eine Besonderheit stellt der Beck'sche Onlinekommentar zum BGB dar, der nicht originär als Onlinekommentar entwickelt wurde. Hier wurden die Autoren des Bamberger/Roth, BGB (siehe oben S. 510) gewonnen, ihr Werk in einen Onlinekommentar umzuwandeln. Dies war kein ganz einfaches Unterfangen, da die Autoren ja nicht mit der Prämisse in das Werk eingetreten sind, vierteljährliche Aktualisierungen vornehmen zu müssen. Am 24. Mai 2006 ging gleichwohl die erste Edition des BeckOK BGB ans Netz.

In den Folgejahren wurde der Kreis der Onlinekommentare zügig erweitert, so dass mit 28 Onlinekommentaren derzeit alle wesentlichen Rechtsgebiete des Bürgerlichen Rechts, des Strafrechts und des Öffentlichen Rechts abgedeckt sind. Besondere Hervorhebung verdienen dabei noch der Beck'sche Onlinekommentar zur Strafprozessordnung, der unter der Herausgeberschaft des Richters am Bundesgerichtshof Jürgen-Peter Graf erschienen ist, sowie die Onlinekommentare zu den zentralen Gebieten des Öffentlichen Rechts wie Epping/Hillgruber, Grundgesetz, Posser/ Wolff, Verwaltungsgerichtsordnung, und Bader/Ronellenfitsch, Verwaltungsverfahrensgesetz. Spät, aber deswegen nicht minder erfolgreich wurde das Programm der Beck'schen Onlinekommentare 2011 um einen Kommentar zur Zivilprozessordnung mit den Herausgebern Rechtsanwalt am BGH Professor Volkert Vorwerk und Professor Christian Wolf veröffentlicht. Ergänzt wurden die Beck'schen Onlinekommentare durch die Reihe der Beck'schen Online-Formulare zu allen Rechtsgebieten. 
https://doi.org/10.17104/9783406684883-471, am 26.04.2023, 11:29:42

Open Access - (c) EY EY - http://www.beck-elibrary.de/agb 